\title{
Crack tip field of a double-network gel: Visualizing covalent bond scission by mechanoradical polymerization
}

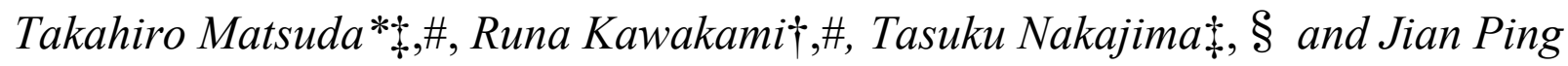
Gong*ะ,§, ๆ

†Faculty of Advanced Life Science, Hokkaido University, N21W11, Kita-ku, Sapporo 001-0021, Japan. †Graduate School of Life Science, Hokkaido University, N21W11, Kita-ku, Sapporo 0010021, Japan. §Soft Matter GI-CoRE, Hokkaido University. ๆInstitute for Chemical Reaction Design and Discovery (WPI-ICRD), Hokkaido University, N21W10, Kita-ku, Sapporo 0010021, Japan.*e-mail: tkhr.matsuda@gmail.com, gong@sci.hokudai.ac.jp \#These authors contributed equally. 


\section{Table of Contents (TOC) graphic (For Table of Contents use only)}

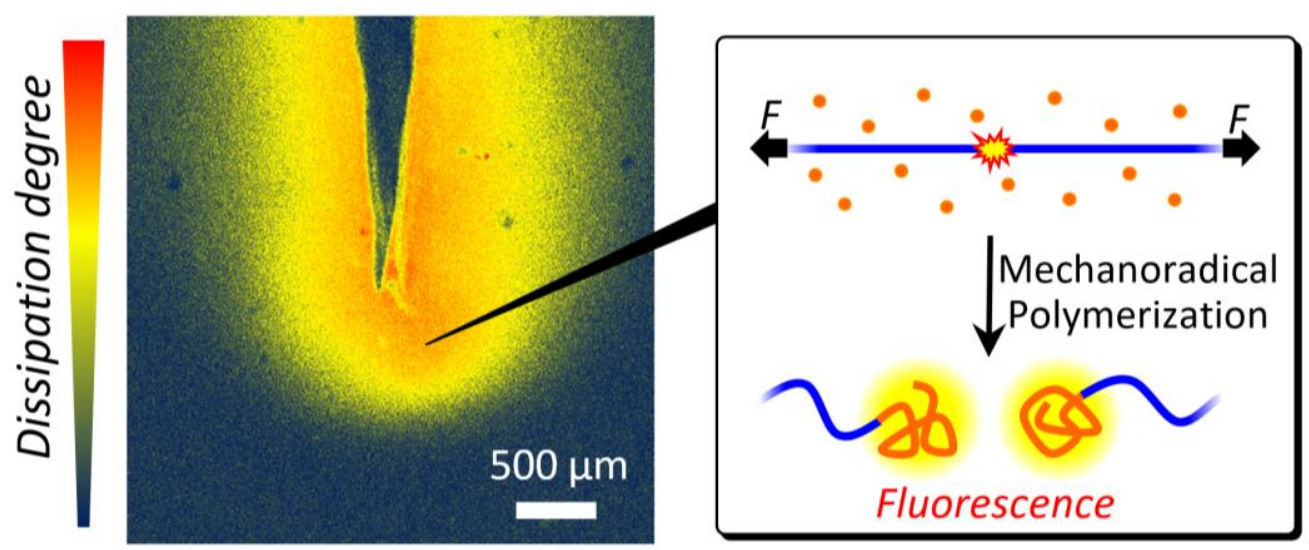

\section{ABSTRACT}

Quantitative characterization of the energy dissipative zone around the crack tip is the central issue in fracture mechanics of soft materials. In this research, we present a mechanochemical technique to visualize the bond scission of the first network in the damage zone of tough double-network hydrogels. The mechanoradicals generated by polymer chain scission are employed to initiate polymerization of a thermoresponsive polymer, which is visualized by a fluorophore. This technique records the spatial distribution of internal fracturing from the fractured surface to the bulk, which provides the spatial profiles of stress, strain, and energy dissipation around the cracktip. The characterized results suggest that, in addition to the dissipation in relatively narrow yielded zone which is mostly focused in the previous works, the dissipation in wide pre-yielding zone and the intrinsic fracture energy have also significant contribution to the fracture energy of a DN gel. 


\section{INTRODUCTION}

Mechanically robust soft materials attract significant interests not only for fundamental science $^{1-5}$ but also for the requirements in emerging applications including medical implants, ${ }^{6,7}$ soft robots ${ }^{8,9}$ and wearable soft electronics. ${ }^{10,11}$ For the practical application to ensure the mechanical reliability, toughness, i.e. crack resistance of the materials, is crucially important along with a high strength and an appropriate stiffness. Recent studies on soft-matter fracture mechanics indicates that the energy dissipation in the namely process zone around the crack tip is important to understand the fracture energy of the soft materials. ${ }^{2,12,13}$ The toughness of the soft materials are typically characterized as the fracture energy $\Gamma_{\mathrm{c}}$ with the unit of $\mathrm{J} / \mathrm{m}^{2}$ that represents the energy required to advance a crack to form the unit area of fractured surface. On the tough soft materials, $\Gamma_{\mathrm{c}}$ is considered comprising two contributions, ${ }^{2,13}$

$$
\Gamma_{c}=\Gamma_{0}+\Gamma_{\text {diss }}
$$

where $\Gamma_{0}$ represents the intrinsic fracture energy directly related to the material separation at the crack tip, and $\Gamma_{\text {diss }}$ represents the dissipation that takes place in the process zone, whose size is typically from sub-micrometer to centimeters depending on the specific materials, owing to viscoelastic dissipation as an example. For toughened soft materials, $\Gamma_{\text {diss }}$ is considered much larger than $\Gamma_{0}$ so that the overall fracture energy $\Gamma_{\mathrm{c}}$ can be approximated as $\Gamma_{\mathrm{c}} \approx \Gamma_{\text {diss. }}$ Therefore, elucidating the dissipation mechanism in the process zone and its quantitative understanding is the key to investigate the soft matter toughness.

Among the efforts of making tough soft materials including hydrogels and elastomers, a double-network (DN) concept ${ }^{14}$ provides an effective and general strategy, applicable to 
polymeric networks made from various chemistries. ${ }^{15-20}$ Specifically, a DN material comprises two contrasting polymer networks; one is the brittle and sparse network and the other is the stretchable and concentrated network. ${ }^{15}$ The brittle and the stretchable networks are customary called as the first and the second networks, respectively, according to their sequence in the twostep synthesis. For a typical DN hydrogel, the concentrations of the first and the second networks are $1-3 \mathrm{wt} . \%$ and $10-20 \mathrm{wt} . \%$, respectively, and the rest is water. Even possessing $80-90 \mathrm{wt} . \%$ water, a DN gel shows remarkably high mechanical performance such as elastic modulus of $10^{-1}-10^{1} \mathrm{MPa}$, tensile strain at break of $>1000 \%$ and tensile nominal stress at break of $10^{-1}-10^{1}$ MPa in typical. ${ }^{15,21,22}$ A DN gel shows extremely high fracture toughness of $100-4000 \mathrm{~J} \mathrm{~m}^{-2}$ that is one- or two-order magnitude higher than that of the conventional hydrogels. ${ }^{15,21,23,24}$ Its high resistance of fatigue fracture has also been reported..$^{25}$

The toughening mechanism of DN materials has been substantially investigated. ${ }^{15,19,26-33}$ The findings of yielding accompanying necking, ${ }^{26,34}$ strain rate-insensitive stress-strain relation and irreversible mechanical hysteresis ${ }^{26,27,31}$ in DN hydrogels gave the essential insight, that is, the scission of the covalent bonds of the first network strands, referring as internal fracturing. ${ }^{15,35,36}$ When a DN gel is stressed, the brittle first network breaks while the stretchable second network maintains the integrity of the material. The internal fracturing suggests that the toughening mechanism is attributed to the formation of a damage zone $e^{28-30}$ around the crack tip where the stress is highly concentrated, similar as Dugdale plastic zone of metals and local crazing of glassy polymers. In the damage zone, plenty of mechanical energy, $\Gamma$ diss in Eq. (1), is dissipated prior to the material separation due to crack progress. Therefore, quantitative characterization of the $\Gamma_{\text {diss }}$ due to internal fracturing in the damage zone is one of the central issues in fracture mechanics of tough DN materials. 
Such toughening mechanism attributed to the damage zone of the DN gels is first modeled theoretically by Brown and Tanaka. ${ }^{28,29} \mathrm{Yu}$ et al. experimentally observed the damage zone with the thickness of several hundreds $\mu \mathrm{m}$ using a 3D violet laser scanning microscope. ${ }^{31}$ Observed by a phase contrast optical microscope as well as the 3D violet laser scanning microscope, Liang et al. have then distinguished three regions (hardened zone, yielded zone and pre-yielding zone) in the damage zone of a thin $(100 \mu \mathrm{m}) \mathrm{DN}$ gel. ${ }^{37}$ However, these optical methods have some limitations. The first limitation is that swelling treatment after the tearing is required to enhance the scattering intensity of the light. Because of the re-swelling, the observed damage-zone thickness $h$ is overestimated. Second, these methods could not quantitatively detect the degree of energy dissipation that is a function of position from a crack tip. Moreover, as the method is based on the light scattering, it can only apply to samples that form strong heterogeneity of proper size scale by the internal fracture. In fact, such damage zone with difference light scattering was not detected in some DN gels with different compositions (unpublished). Therefore, new characterization technique suitable for the DN gels are demanded.

In the recent decades, a research field of mechanochemistry, i.e. chemical transformation induced by mechanical stress, has been increasingly growing. ${ }^{38-41}$ Most recently, such mechanochemical technique has been utilized to investigate the mechanics of polymeric materials. ${ }^{19,42-52}$ For example, the scission of the covalent bonds and the force in the polymeric strand in multiple-network elastomers were visualized in-situ by light emission using a dioxetane mechanophore and by color change using a spiropyran mechanophore, respectively, incorporated in the first network crosslinker. ${ }^{19,46,52}$ 
Herein, we propose a novel technique to visualize the bond scission and to characterize the wide damage zone around the crack tip of DN hydrogels, inspired by our recent finding ${ }^{36}$. The technique is based on the combination of the mechanoradical polymerization and fluorescent microscopic technique as conceptually illustrated in Figure 1. Specifically, the internal fracture of DN hydrogels generates large amount of mechanoradicals at the ends of the broken first network strands. ${ }^{36}$ These chemically active mechanoradicals are used to initiate the polymerization of pre-loaded monomer, $N$-isopropylacrylamide, which forms temperatureresponsive polymer, poly( $N$-isopropylacrylamide) (PNIPAAm).${ }^{53}$ The formation of PNIPAAm chains tethered at the broken ends of the first network strands is visualized by pre-loaded fluorescent molecule, 8-anilino-1-naphthalenesulfonic acid (ANS), that exhibits strong fluorescence in the hydrophobic environment. ${ }^{54,55}$ Because PNIPAAm becomes hydrophobic above its lowest critical solution temperature (LCST) at ca. $30-35^{\circ} \mathrm{C},{ }^{53}$ ANS exhibits strong fluorescence in the damaged region above the LCST. Therefore, by using a laser scanning confocal microscope (LSCM), the two- and three- dimensional images of the damage zone can be visualized without re-swelling treatment of the samples. Furthermore, the degree of the internal fracture in the damage zone can be quantitatively studied from the fluorescence intensity distribution because the amount of PNIPAAm increases with the concentration of mechanoradicals that corresponds to the degree of bond scission. An advantage of this technique is broad applicability for DN hydrogels with varied chemistry, without need of mechanophores incorporated to the polymer network which requires special synthesis and can influence the network structure and mechanical properties of the sample. ${ }^{19}$ Because it is based on posterior observation of recorded mechanical history, this method can in principle also characterize the high-speed fracture and complicated deformation of materials, which are often difficult to be 
detected by real-time observations. In this paper, we first visualize the damage zone of a DN gel with this method; then, we extract the profiles of stress, strain, and dissipated energy density from the quantitative fluorescent results. Accumulated energy dissipation in the damage zone is also discussed.

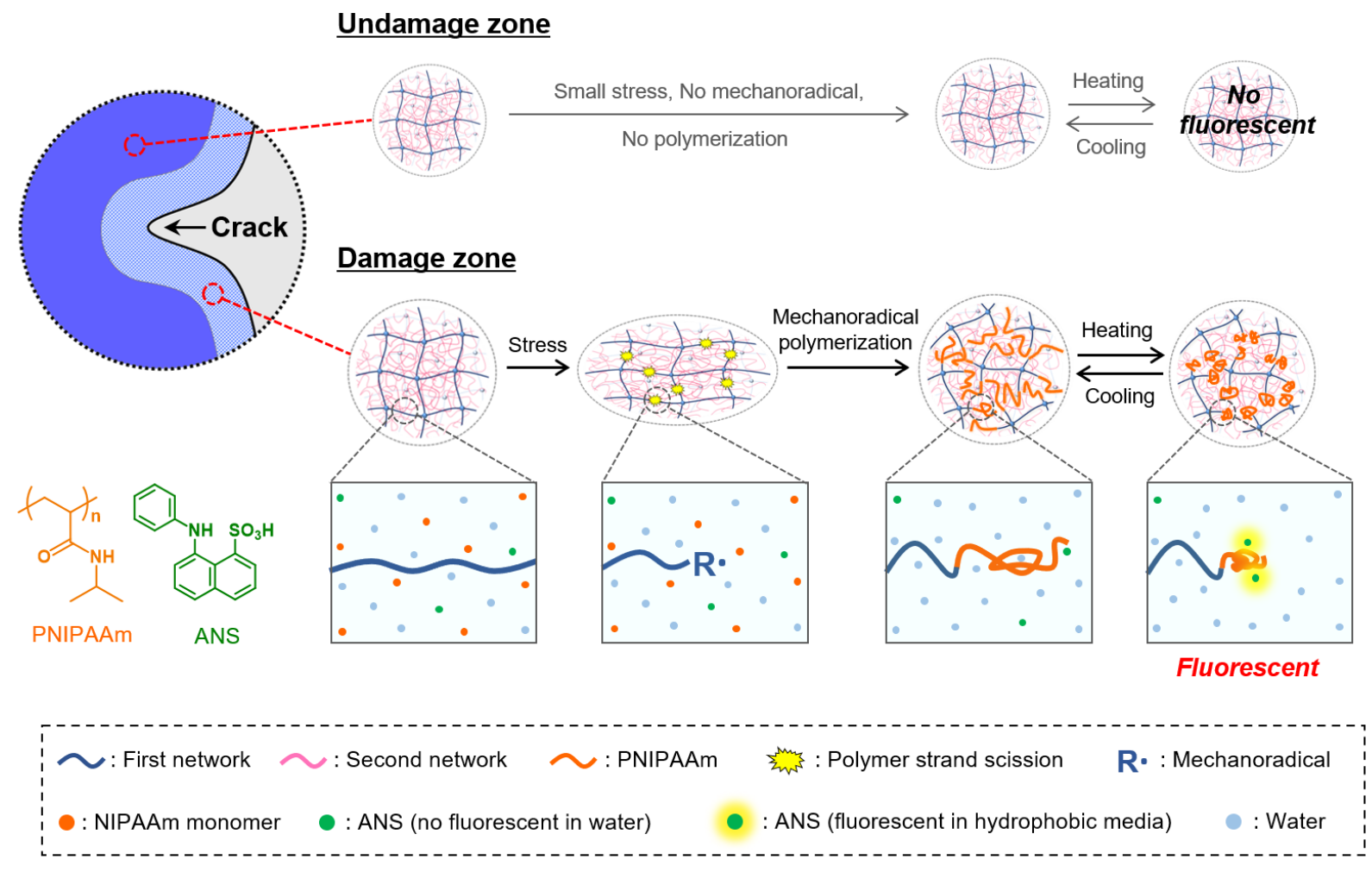

Figure 1. Conceptual scheme of visualizing the damage zone where internal fracturing of the first network occurred around the crack tip in the DN gels. In the damage zone, the mechanoradicals generated by polymer strand scission initiate the polymerization of NIPPAm to form PNIPAAm, a thermo-responsive polymer showing LCST-type micro-phase separation. Because the fluorescent molecule ANS exhibits strong fluorescence only in hydrophobic environment, the damaged region is visualized when observed at a temperature above the LCST of the PNIPAAm while the undamaged region hardly shows fluorescence. The fluorescence images and spatial distributions of fluorescence intensity around the crack tip can be obtained by a laser scanning confocal microscope (LSCM). 


\section{EXPERIMENTS}

Materials. 2-Acrylamido-2-methylpropane sulfonic acid sodium salt (NaAMPS, 49.8 wt.\% aqueous solution) was provided by Toagosei. $N, N^{\prime}$-Methylenebis(acrylamide) (MBAA), 2oxoglutaric acid and $N$-isopropylacrylamide (NIPAAm) were purchased from FUJIFILM Wako Pure Chemical Corporation. Acrylamide (AAm) was purchased from Junsei Chemical. 8Anilino-1-naphthalenesulfonic acid (ANS) was purchased from Tokyo Chemical Industry. Acetone was purchased from Kanto Chemical. All materials except for AAm were used as received, and AAm was recrystallized from acetone once before use.

Synthesis of DN gels. DN gels were synthesized following previous report. ${ }^{21,36}$ First, poly(2acrylamido-2-methylpropane sulfonic acid sodium salt) (PNaAMPS) single-network gels were synthesized as the first network. An aqueous solution of 1.0 M NaAMPS, 3.0-3.5 mol\% MBAA and $1 \mathrm{~mol} \%$ 2-oxoglutaric acid was prepared, where the concentration denoted as $\mathrm{mol} \%$ is the molar percentage with respect to the monomer. For quantitative discussion to compare tearing test and tensile test, MBAA concentration is fixed as $x_{1}=3.0 \mathrm{~mol} \%$. The solution was poured in a glass mold made of two flat glass plates separated with silicone rubber spacer $(1.0 \mathrm{~mm}$ in typical) in an argon glove box. The solution in the mold was irradiated with UV light (365 nm, 4

$\mathrm{mW} \mathrm{cm}{ }^{-2}$ ) for 8 hours in the glove box to proceed the radical polymerization, resulting in a PNaAMPS single-network gel sheet. Afterwards, the PNaAMPS gel was immersed in a second network precursor solution for more than 1 day at $10^{\circ} \mathrm{C}$ to swell the gel and introduce the second-network monomers to the gel. The second-network precursor aqueous solution comprises 2.0-4.0 AAm, 0.01-0.02 mol\% MBAA and $0.01 \mathrm{~mol} \%$ 2-oxoglutaric acid. For quantitative discussion to compare tearing test and tensile test, AAm and MBAA concentrations are fixed as 
$C_{2}=4.0 \mathrm{M}$ and $x_{2}=0.02 \mathrm{~mol} \%$, respectively. The swollen gel was sandwiched between two flat glass plates, moved to the argon glove box, and then irradiated with UV light for 9 hours in the glove box to synthesize the second PAAm network in the presence of the first PNaAMPS network. The synthesized DN gel was immersed in large volume of deionized water for at least 1 day to remove unreacted reagents and make the gel equilibrium-swollen state in water.

Tensile test for mechanical characterization. To characterize the tensile mechanical property of a DN gel, we conducted uniaxial tensile test and uniaxial cyclic tensile test. In the uniaxial tensile test, dumbbell-shaped DN gels (standardized to JIS-K 6251-7; 12-mm gauge length, 2mm width and $3.1 \mathrm{~mm}$ thickness) were uniaxially stretched with a tensile tester (INSTRON 5965 , Instron Co.) at a cross-head velocity of $100 \mathrm{~mm} \mathrm{~min}^{-1}$ (nominal strain rate of $\sim 0.1 \mathrm{~s}^{-1}$ ). The stress $\sigma$ was characterized as nominal stress that is the measured force divided by original cross-sectional area. The strain $\varepsilon$ was measured using a non-contacting video extensometer (AVE, Instron Co.). Elongation ratio $\lambda$ is calculated as $\lambda=\varepsilon+1$. With the same experimental condition, cyclic tensile test at varied strain $\varepsilon$ was also performed to characterize the dissipated mechanical energy density $U_{\text {diss }}$ as a function of $\varepsilon$ from mechanical hystereisis. ${ }^{35,36}$

\section{Tearing test for mechanical characterization.}

To characterize the tearing fracture energy $T_{\mathrm{c}}$ of the $\mathrm{DN}$ gel, trouser-type tearing fracture test was carried out. ${ }^{2,56,57}$ A trouser shaped sample (thickness $t=3.1 \mathrm{~mm}$, full width $8.0 \mathrm{~mm}$, each leg's width $w=4.0 \mathrm{~mm}$, full length $\sim 50 \mathrm{~mm}$, and initial cut length $\sim 20 \mathrm{~mm}$, see Figure S1) was torn at the cross-head velocity of $100 \mathrm{~mm} \mathrm{~min}^{-1}$ with a tensile tester (INSTRON 5965, Instron Co.). During the tearing, elongation of the leg was measured using video extensometer (see Figure S1). ${ }^{20}$ The tearing fracture energy $T_{\mathrm{c}}$ was calculated as 


$$
T_{\mathrm{c}}=\frac{2 F_{\mathrm{c}} \lambda_{\mathrm{c}}}{t}-2 w W_{\mathrm{c}}
$$

where $F_{\mathrm{c}}, \lambda_{\mathrm{c}}$ and $W_{\mathrm{c}}$ are the average force, the average elongation ratio of the leg, and the average strain energy density of the leg, respectively, during the crack propagation.

Tensile and tearing tests for fluorescent measurement. To induce the mechanoradical polymerization in the $\mathrm{DN}$ gels during mechanical tests (tensile and tearing tests), these mechanical tests were carried out in an argon glove box (oxygen concentration of $<50 \mathrm{ppm}$ in typical) to avoid inhibition of radical polymerization by oxygen. ${ }^{36}$ First, the DN gels cut with testing shapes (dumbbell and trouser shapes) were immersed in a filtered aqueous solution of 1.0 M NIPAAm and $200 \mathrm{mg} / \mathrm{L}$ ANS, in typical, for 1 day in an argon glove box at $\sim 15^{\circ} \mathrm{C}$. Then the sample containing NIPAAm and ANS was mounted to a tensile tester (MCT-2150, A\&D Co.) and stretched or torn in the glove box at $\sim 15^{\circ} \mathrm{C}$. The testing velocity and sample geometry were the same as mechanical characterization shown above. In the tensile test, the imposed maximum strain was measured by applying two dots on the gel and measuring the distance between the dots by caliper. After the stretching or tearing, the gel was wrapped with plastic film (Saran wrap) to avoid drying, and then stored in the glove box overnight ( $\sim 18$ hours) to proceed the mechanoradical polymerization. The samples were then taken from the glove box, and the fluorescent property was characterized.

Fluorescent spectroscopy. Fluorescent spectrum was obtained using fluorescent spectrometer (FP-6600, Jasco Co.) equipped with epifluorescence unit (EFA-383, Jasco Co.). The excitation wavelength was $402 \mathrm{~nm}$. 
Fluorescent observation using laser scanning confocal microscope (LSCM). Fluorescent microscopic measurement was carried out using a laser scanning confocal microscope (LSCM) (Nikon A1 Rsi and Ti-E, Nikon Co.) equipped with a Plan Fluor x4 objective lens (NA 0.13, Nikon Co.). The excitation laser wavelength was $402.5 \mathrm{~nm}$. Fluorescent emission was collected in a wavelength range of $425-475 \mathrm{~nm}$. In all measurements, sample was put on a glass-bottom dish (3970-035-SK, IWAKI \& Co.) and maintained at $42^{\circ} \mathrm{C}$ (above LCST of PNIPAAm) by using a stage-top incubator (INUBG2H-TIZB, Tokai Hit Co.). For the qualitative observation (Figures 3, 5, 6, S3 and S4), the excitation laser intensity was tuned to appropriate values to show clear images. For the quantification purpose (Figures 8-10), the same laser intensity was used for all the measurements. 


\section{RESULTS and Discussion}

\section{Mechanoradical polymerization and fluorescent emission}

First, we investigated the mechanoradical polymerization of NIPAAm in a stretched DN gel and the resulting fluorescent behavior of the sample. All measurements were carried out at relaxed (stress-released) state of the gels. Hereafter, the "unstretched" gel denotes a sample to which no stretch was applied (strain $\varepsilon=0$ ), and "stretched" gel denotes a sample to which a prescribed stretch $(\varepsilon>0)$ was applied and then the stretch was released so that the sample length almost returned to the original length at the relaxed state. Figure 2a shows the optical images of unstretched $(\varepsilon=0)$ and stretched $(\varepsilon \approx 4)$ DN gel fed with NIPAAm and ANS observed at $45{ }^{\circ} \mathrm{C}$. The unstretched DN gel is transparent, while the stretched one is opaque. The opaque appearance of the stretched sample indicates that NIPAAm in the DN gel was polymerized by the mechanoradicals and the synthesized PNIPAAm underwent micro-phase separation above the LCST. The monomer conversion was determined as approximately $40 \%$ in this typical case (see Figure S2). The stretched DN gel also exhibits strong fluorescence, which further confirms the formation of the PNIPAAm in the gel (Figure 2b). We here measured the fluorescent spectrum at $45^{\circ} \mathrm{C}$ using a fluorescent spectrometer with an excitation wavelength of $402 \mathrm{~nm}$. As shown in Figure 2b, the stretched DN gel exhibits strong fluorescence at around $470 \mathrm{~nm}$, while the unstretched DN gel shows negligible fluorescence. This result demonstrates that we can detect internal fracturing in deformed DN gels by fluorescence.

Next, we observed the damage zone around the fractured surface of a DN gel macroscopically. Figure 2c shows the optical images of a trouser-shaped DN gel torn by hand. As expected, only the region around the torn surface becomes opaque under visible light (Figure 2c(i)) and exhibits 
strong fluorescence under UV light (Figure 2c(ii)). These results clearly indicate that internal fracturing has occurred around the crack tip during the tearing of the sample. The thickness of the damage zone $h$ from the fractured surface to the bulk is roughly $\sim 1 \mathrm{~mm}$, which is close to the value observed in previous reports. ${ }^{31,37}$

(a) (i) unstretched

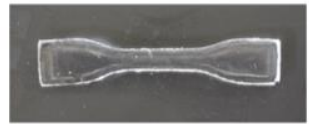

(b)

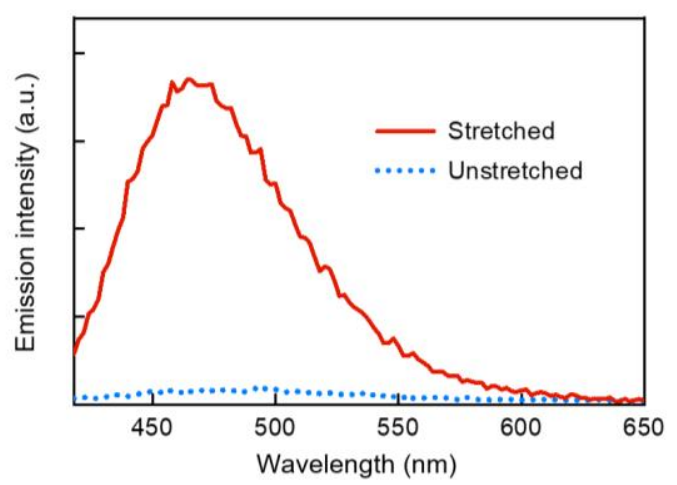

(c) (i) Torn, under visible light

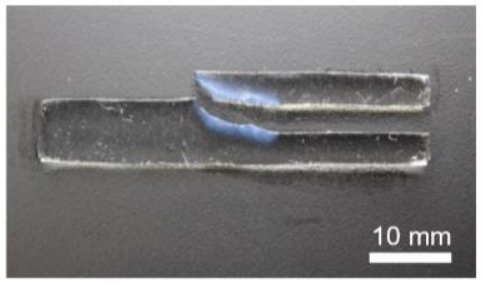

(ii) Torn, under UV light

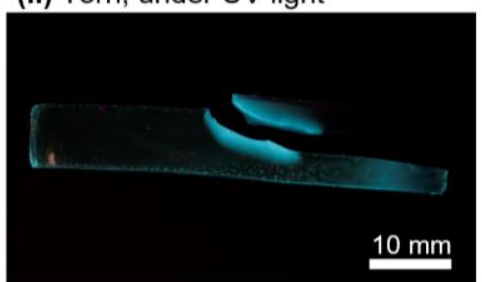

Figure 2. (a) Optical images of (i) unstretched and (ii) stretched $(\varepsilon \approx 4)$ DN gels fed with NIPAAm and ANS under visible light observed at $45^{\circ} \mathrm{C}$. (b) Fluorescent spectra (excitation: $402 \mathrm{~nm}$ ) of the corresponding DN gels at $45^{\circ} \mathrm{C}$. (c) Optical images of a torn DN gel fed with NIPAAm and ANS observed at $45^{\circ} \mathrm{C}$ under (i) visible light and (ii) UV light (365 nm). 


\section{Fluorescent imaging by LSCM}

Using the laser scanning confocal microscope (LSCM), we visualize the two- and threedimensional fluorescent images of a torn sample. Figure 3a shows the optical image of the trouser-shaped specimen torn for several millimeters. A strongly opaque region around the crack tip, consistent with Figure 2c(i), is observed. Figure 3b shows a two-dimensional fluorescent image taken at a depth $5 \mu \mathrm{m}$ from the sample surface. A fluorescent region with arc-shape in front of the crack tip and strip-shape along the fractured surface, corresponding to the damage zone, is observed. Figure 3c shows a three-dimensional image of the crack tip from the top surface to the bottom surface of the gel. The image is constructed from the two-dimensional images measured at different depths from the top surface.

(a)

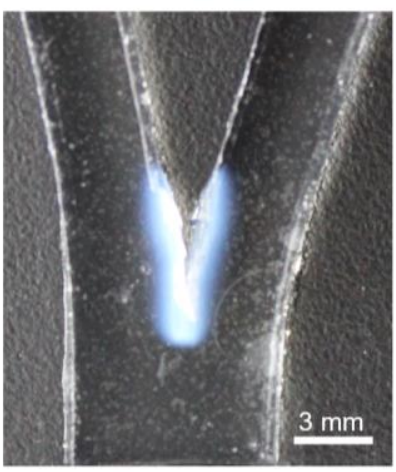

(b)

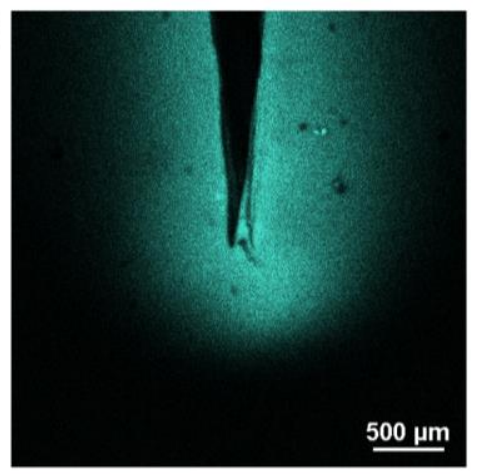

(c)

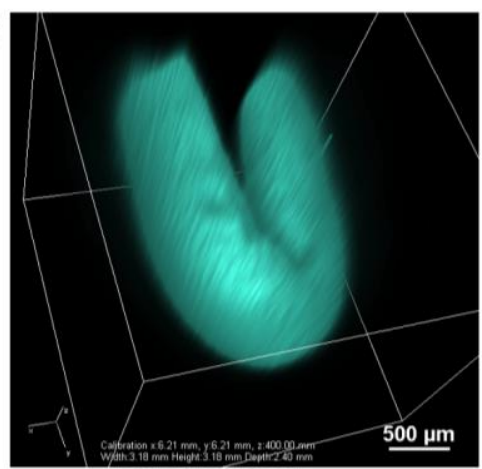

Figure 3. (a) An optical image, and (b) two-dimensional and (c) three-dimensional fluorescent images of laser scanning confocal microscopy (LSCM) around the crack tip of a torn DN gel fed with NIPAAm and ANS observed at $42^{\circ} \mathrm{C}$. 


\section{Fluorescent intensity profile}

Next, we measured the fluorescent intensity profile in the damaged zone using a trouser shaped specimen (Figure 4). To facilitate the later discussion, we set the coordinate for one fractured piece in the relaxed state as shown in Figure 4 (right). The fractured surface is set in xy plane with $z=0$, the direction crack advanced is set along the $\mathrm{x}$-axis, $y$ is set as zero on one of the sample surface, and the z-axis takes positive values inside the sample. The two-dimensional observation with LSCM was performed in the xz plane at a depth $y=5 \mu \mathrm{m}$ from the gel surface (see Figure 4). The fluorescent intensity profile, $I(z)$, was obtained by line scan, at a step of 6.2 $\mu \mathrm{m}$, along the $\mathrm{z}$-direction from the fractured surface $(z=0)$ to the bulk $(z \sim 2000 \mu \mathrm{m})$. The raw data contained relatively strong noise, which might originate from the factors of the equipment such as detector sensitivity and/or the micro-scale inhomogeneity of the internal fracturing. Hence, we show moving-averaged data over 9 data points, which gives an average over a length scale of $\sim 50 \mu \mathrm{m}$ in $\mathrm{z}$-direction, in all the figures shown in the main text (see Figure $\mathbf{S 3}$ for detail). This length taking average $(\sim 50 \mu \mathrm{m})$ is much smaller than the size of damage zone under studying. A typical LSCM image and the corresponding fluorescent intensity profile of a torn DN gel are shown in Figures 5a(i) and 5b(i), respectively. The fluorescent intensity, reflecting the degree of polymer strand scission, shows a gradient distribution in the damage zone. It increases gradually towards the fractured surface and reaches the maximum almost at the surface. The fluorescent intensity distribution records the mechanical history around the crack tip reflecting the energy dissipation, which relates to the maximum stress and strain experienced by the specimen during the tearing. 
From the intensity profile, we determined the damage-zone thickness. Because the fluorescent profile is an attenuation function approaching to the intensity $I \approx 0$, the end point of the damaged zone was determined for convenience as the point where the fluorescent intensity decreased to $5 \%$ of the maximum intensity $I_{\max }$ closed to the fractured surface, $I(h) / I_{\max }=0.05$ (see Figure S4). The damage-zone thickness thus defined is $h=1700 \mu \mathrm{m}$ for this torn DN gel (Figure 5b(i)).

We notice that the damage zone profile strongly depends on the fracture method. When the DN gel was cut with scissors and razor blade, the damage-zone thickness decreased to $h=$ $610 \mu \mathrm{m}$ and $510 \mu \mathrm{m}$, respectively (Figures $\mathbf{5 b ( i i )}$ and (iii)), while their maximum intensity $I_{\max }$ near the fractured surface are almost the same with the torn sample. Since the observations were performed at the same experimental condition, this result shows that the applied stress and deformation near the cut surface hardly depend on the fracture method while the stress and strain fields away from the crack tip is dominated by the fracture method. It may be worth mentioned in some practices that internal damage in the range of $\sim 100 \mathrm{~s} \mu \mathrm{m}$ possibly presents when a DN gel is cut even by razor blade. In the following discussion, we focus on the characterization of samples fractured by tearing of trouser-shaped sample as illustrated in Figure 4.

The spatial resolution of this method based on mechanoradical polymerization and fluorescent measurement can be limited by two factors; the LSCM equipment and the size of PNIPAAm tethered to the partially broken PNaAMPS network. The theoretical resolution of the LSCM is $\sim 1 \mu \mathrm{m}$ along the scanning direction (z-direction), considering the objective lens (numerical aperture 0.13) used under air (refractive index 1.0) and excitation wavelength (402 $\mathrm{nm})$. On the other hand, the size of the PNIPAAm chain is estimated as follows. For the estimation, we assumed that the maximum possible polymerization degree $N$ of PNIPAAm is as 
high as $N=10^{6}$ (molecular weight of $\left.10^{8} \mathrm{~g} \mathrm{~mol}^{-1}\right)$. In the expected random coiled state below LSCT, the radius of the PNIPAAm chain is roughly approximated as $a N^{1 / 2} \sim 250 \mathrm{~nm}$, where $a$ is the length of monomer unit $(a \approx 0.25 \mathrm{~nm})$. At above LCST during the fluorescent observation, the size of the globule PNIPAAm must be smaller than the coil size. Therefore, the size of a PNIPAAm chain under the observation at above LCST must be smaller than $250 \mathrm{~nm}$. Both the theoretical resolution $(\sim 1 \mu \mathrm{m})$ and the size of a PNIPAAm chain $(<250 \mathrm{~nm})$ are much smaller than the resolution of our 9-points moving-average $(\sim 50 \mu \mathrm{m})$. Therefore, the resolution by the procedure of this work is expected as $50 \mu \mathrm{m}$.

The resolution is confirmed by the observation of the fractured surface of a singlenetwork gel (Figure 6). According to the Lake-Thomas theory, the damage-zone thickness of an elastic single-network gel is considered to be in the order of the mesh size $(\sim 10 \mathrm{~s} \mathrm{~nm})^{58,59}$ that is enough smaller than the expected resolution $(\sim 50 \mu \mathrm{m})$. In this experiment, a PNaAMPS singlenetwork gel fed with NIPAAm monomer was broken by bending because this gel is stiff and very brittle. As shown in Figures 6a and 6b, left leg was broken in argon atmosphere to proceed the mechanoradical polymerization, while right leg was broken in air as control to inhibit the mechanoradical polymerization by oxygen. Observed with LSCM, a clear fluorescent line is found only on the left fracture surface (Figure 6c). This fluorescent is not due to the surface effect (such as drying effect) but comes from the mechanoradical polymerization because such fluorescence was not observed in the right side where mechanoradical polymerization is inhibited. The moving-averaged fluorescent profile (Figure 6d) indicates that the thickness of the fluorescence around the fractured surface is $\sim 60 \mu \mathrm{m}$, which is very close to the expected resolution of $\sim 50 \mu \mathrm{m}$. The result also demonstrates that this technique can be used not only for 
fracture of $\mathrm{DN}$ gels but also for fracture of other materials accompanied with homolytic bond scissions. The spatial resolution should be considered or improved on some applications.

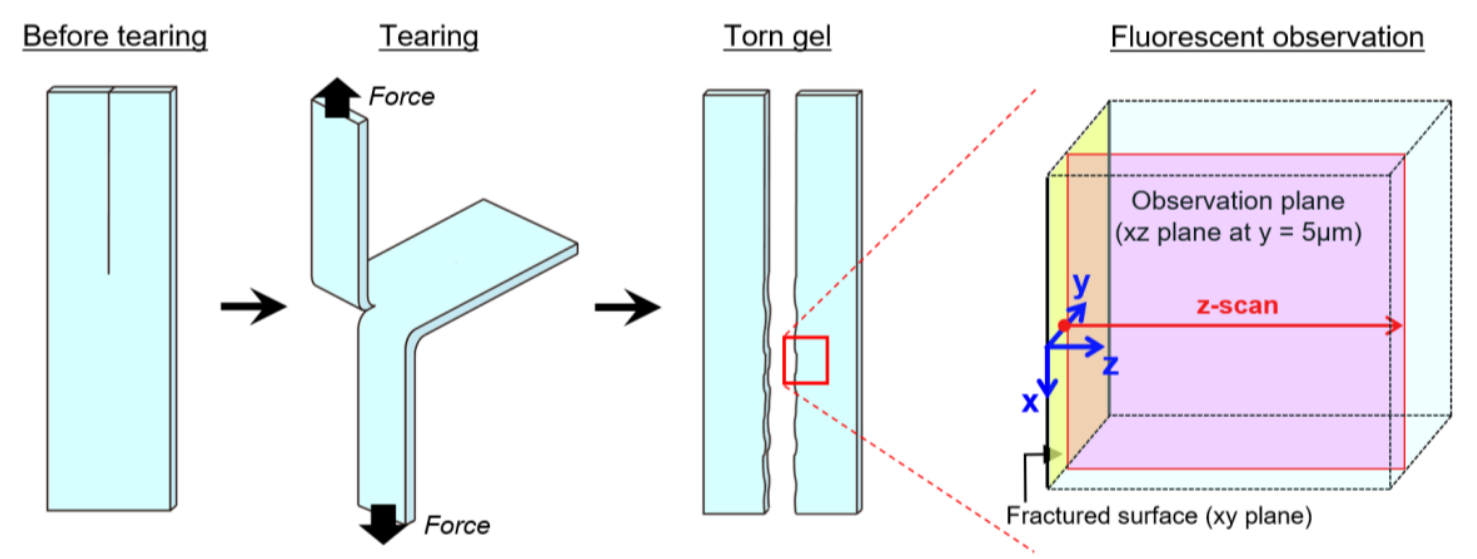

Figure 4. Schematic illustration of the tearing test of DN gels and the observation plane of fluorescent image by LSCM. The fluorescent intensity images were obtained in the xz plane that is vertical to the fractured surface (xy plane), and the line scans were performed along the z-axis $5 \mu \mathrm{m}$ beneath the sample surface. 
Torn

(a)
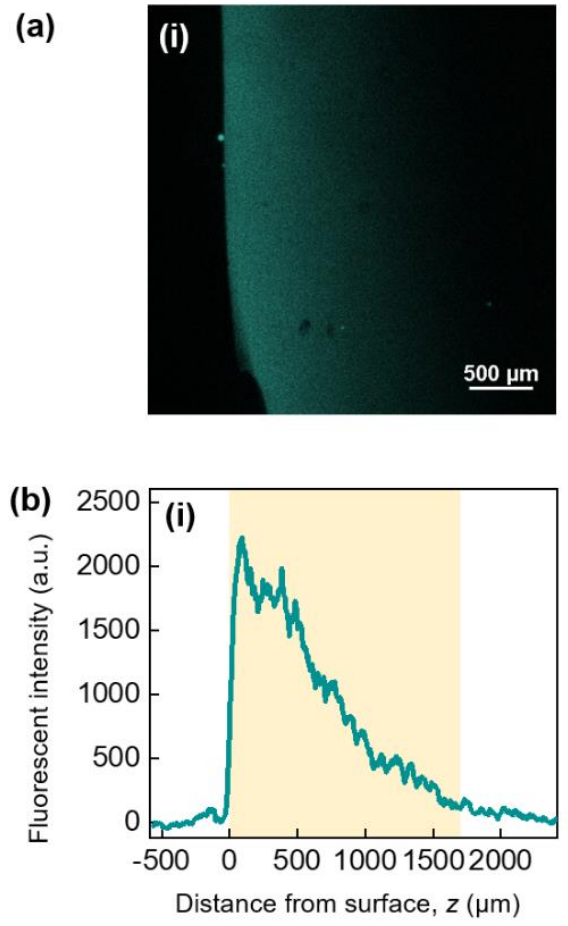

Cut with scissors
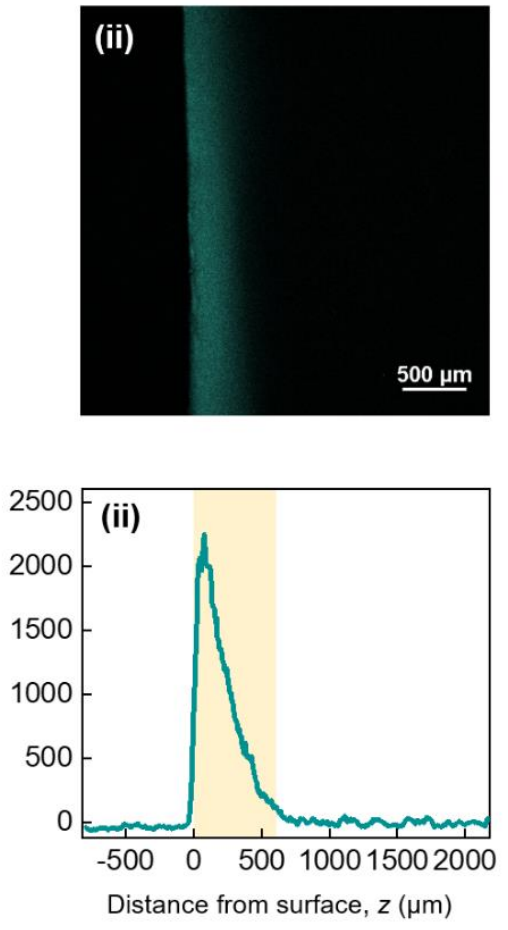

Cut with razor blade

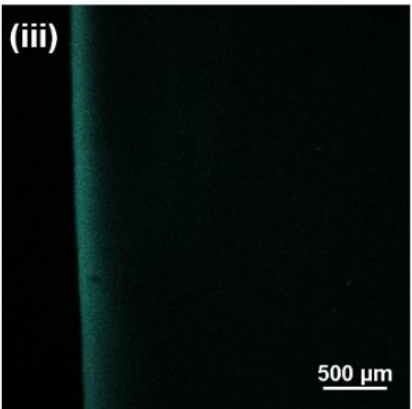

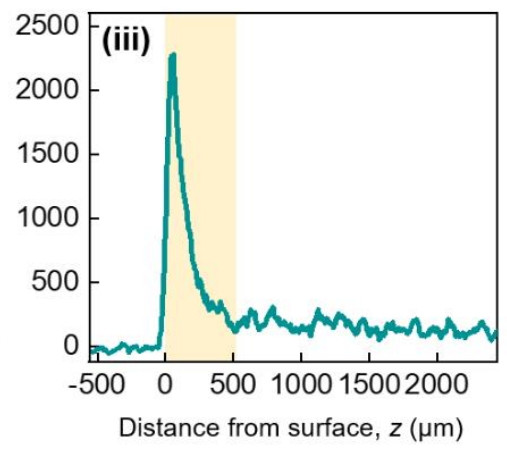

Figure 5. (a) Fluorescent images and (b) line profiles of fluorescent intensity $I(z)$ of the DN gels from fractured surface to bulk. The DN gel was ruptured by different modes, (i) fractured by tearing using trouser-shaped sample (see Figure 4), (ii) cut with scissors, and (iii) cut with a microtome razor blade. Yellow highlighted regions in (b) denote the damage-zone thickness $h$ estimated from the fluorescent intensity $I(h) / I_{\max }=0.05$, where $I_{\max }$ is the maximum intensity close to $z=0$ (see Figure S4). The same experimental conditions were used for these three individual experiments. The profiles in (b) are the moving-averaged data (see Figure S3). 
(a)

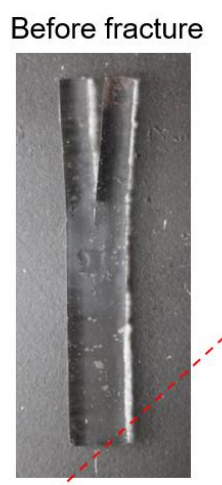

After fracture

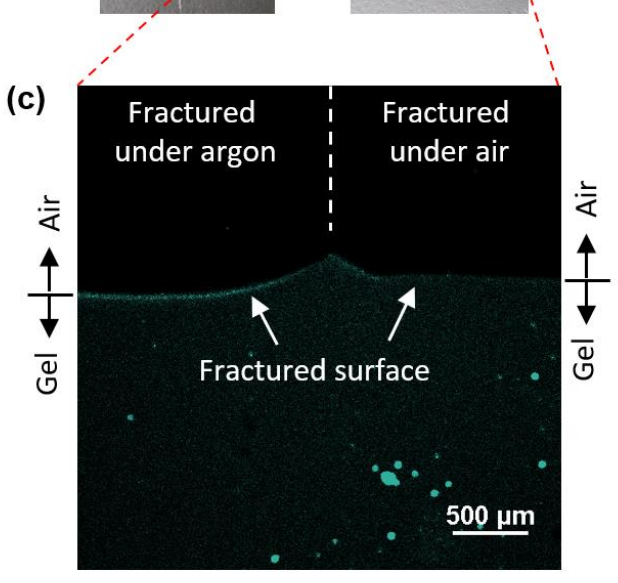

(b)
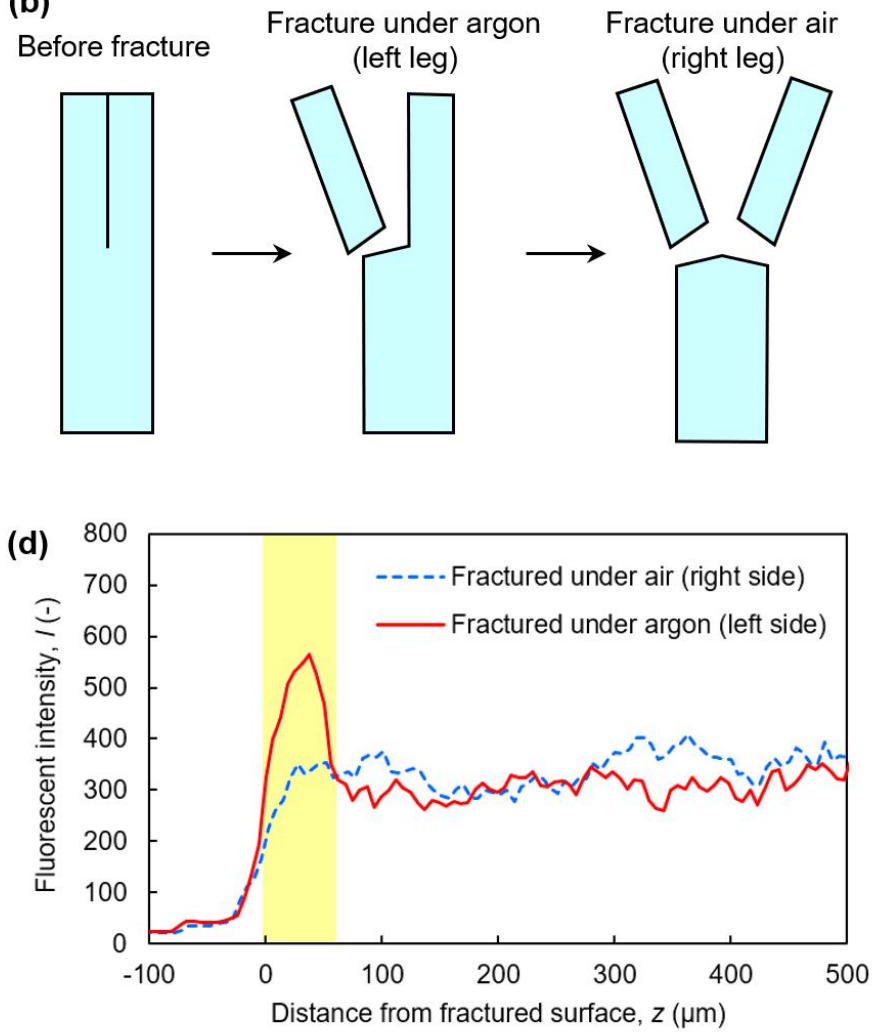

Figure 6. Mechanoradical polymerization around the fractured surface of a PNaAMPS singlenetwork gel. (a) Optical images of the PNaAMPS gel before and after fracturing. (b) Schematic illustration of the fracture method. (c) Fluorescent image taken by LSCM. (d) Fluorescent intensity profiles of the fractured PNaAMPS gel. Fluorescence around the fractured surface was observed only in the left side because the mechanoradical polymerization in the right side was inhibited by oxygen under air. The profiles are the moving-averaged data. 


\section{Damage zone characterization of a typical DN gel}

Next, we intend to estimate the distribution of stress $\sigma$, strain $\varepsilon$, and dissipated energy density $U_{\text {diss }}$ around the crack tip of a DN gel from the recorded fluorescent intensity profiles.

Specifically, we first measure the fluorescent intensity of DN gels uniaxially stretched at various $\varepsilon$ to get fluorescent intensity $I$ as a function of $\varepsilon$. From the tensile stress-strain curve and cyclic tensile test result, we get stress $\sigma$ and energy dissipation $U_{\text {diss }}$ at various strain $\varepsilon$. Using these tensile results and the fluorescent profile $I(z)$ of the fractured sample, we get the $\sigma(z), \varepsilon(z)$, and $U_{\text {diss }}(z)$ profiles in the fractured sample, which reveal the maximum stress and strain, and the dissipated energy distribution around the crack tip during the tearing process. For this analysis, we assume that the deformation of all elements can be approximated as uniaxial tension along the z-axis although the deformation near the crack tip of a sample during tearing must be more complicated. This assumption is based on our following considerations. First, a theory of nonlinear elastic solids indicates that the deformation ahead of the crack is dominated by pure uniaxial tension. ${ }^{60,61}$ With the increase of the distance from the crack tip, the uniaxial tension would gradually change to the pure-shear tension. Even so, it has known that the stress-strain curves of a DN gel in uniaxial and pure-shear deformation are similar. ${ }^{62}$ With this assumption, we use the fluorescent results of uniaxial tensile test to calibrate the stress and strain fields around the crack tip as an approximation.

For the measurement, we used a DN gel (see experimental section), which is slightly different from the DN gels shown above, having the following mechanical features. The DN gel shows yielding and strain hardening phenomena under uniaxial tensile test. Under the tearing test (Figure 7), the DN gel shows stable (i.e. no stick-slip) crack propagation (Figure 7a), and its 
fracture energy is $T_{\mathrm{c}}=906 \mathrm{~J} \mathrm{~m}^{-2}$. Note that strain of the sample's leg far from the crack was very small $(\varepsilon \approx 0.14$, Figure $7 \mathbf{b})$ below the threshold strain $(\varepsilon \approx 0.4)$ to show mechanical hysteresis and fluorescence (shown later in Figures $8 \mathbf{b}$ and 10a), thus effect of the leg stretch on the fluorescent intensity of the torn sample is negligible. Uniaxially stretched samples and a torn sample were measured by LSCM at the same experimental conditions for the quantitative comparison.
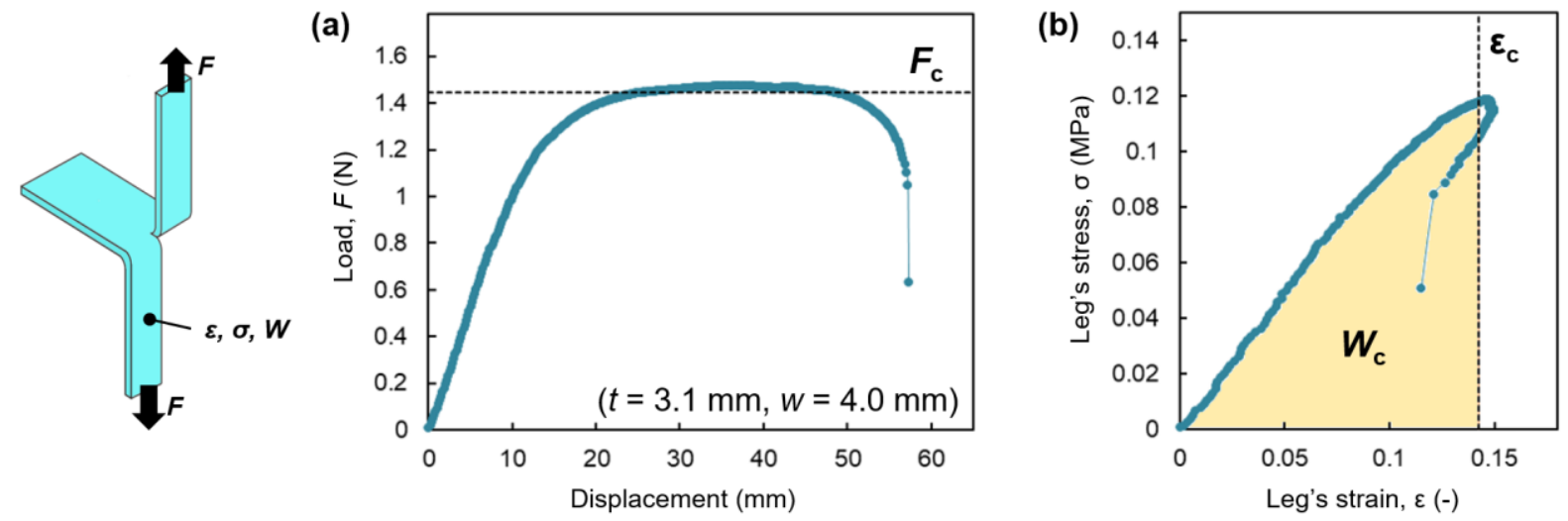

Figure 7. A typical result of tearing fracture test of a trouser-shaped DN gel. (a) Forcedisplacement curve of the specimen and (b) stress-strain curve of a sample leg far from the crack tip. During the stable crack propagation, the average force was $F_{\mathrm{c}}=1.45 \mathrm{~N}$, and the average elongation ratio and energy density of the leg were $\lambda_{\mathrm{c}}=\varepsilon_{\mathrm{c}}+1=1.14$ and $W_{\mathrm{c}}=9.3 \mathrm{~kJ} \mathrm{~m}^{-3}$, respectively, resulting in tearing fracture energy of $T_{\mathrm{c}}=991 \mathrm{~J} \mathrm{~m}^{-2}$ from Eq. (2). From four measurements, $T_{\mathrm{c}}$ of this DN gel is characterized as $906 \pm 62 \mathrm{~J} \mathrm{~m}^{-2}$ with standard deviation. 
(i) Strain profile. Figures $8 \mathbf{a}$ and $8 \mathbf{b}$ display the uniaxial tensile $\sigma-\varepsilon$ curve and the fluorescent intensity $I$ of the DN gel as the function of tensile strain applied, $\varepsilon$, respectively. The inset letters in Figure 8a indicate (A) $\varepsilon=0,(B) \varepsilon=0.5$ (threshold for exhibiting fluorescence), (C) $\varepsilon=1.0$ (pre-yielding region), (D) $\varepsilon=1.6$ (yield point) and (E) $\varepsilon=3.0$ (strain hardening region). This threshold for exhibiting fluorescence $(\mathrm{B}, \varepsilon=0.5)$ almost corresponds to the threshold for showing mechanical hysteresis due to the internal fracturing (shown later in Figure 10). We found that $I$ monotonically increased with $\varepsilon$ (Figure $\mathbf{8 b}$ ), indicating that the degree of internal fracturing (i.e. concentration of mechanoradicals) monotonically increases with applied strain. Indeed, the dissipated mechanical energy density $U_{\text {diss }}$ that characterizes the degree of internal fracturing also monotonically increased with $\varepsilon$ (Figure 10a, discuss later). This is consistent with our previous work that also revealed a monotonic increase of mechanoradical concentration with $\varepsilon$ and $U_{\text {diss. }}{ }^{36}$ 

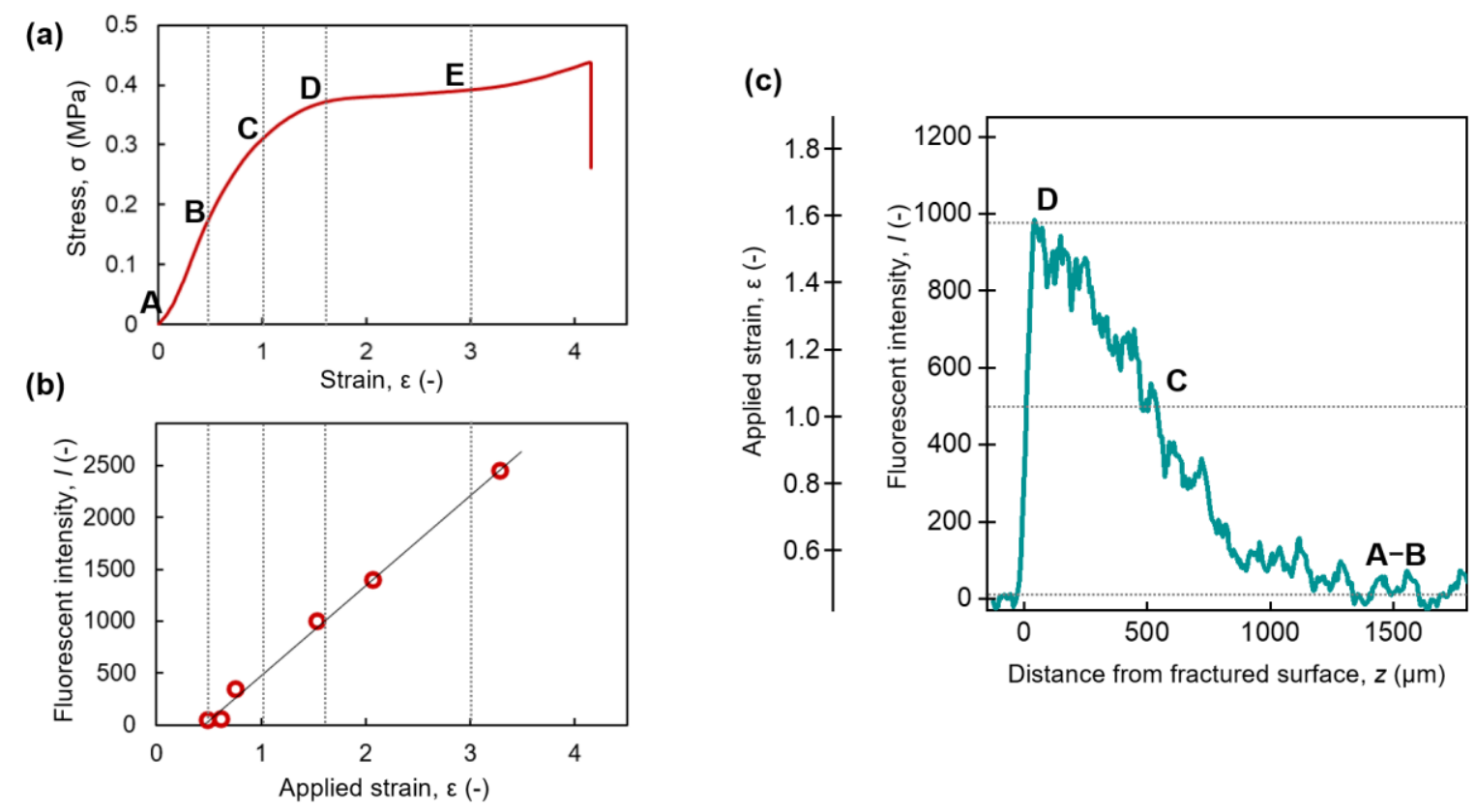

Figure 8. (a) A uniaxial tensile $\sigma-\varepsilon$ curve, (b) $I-\varepsilon$ relation of a DN gel after the uniaxial tensile deformation, and (c) $I$ and estimated $\varepsilon$ profiles of a torn sample from fractured surface to bulk. In (c), $\varepsilon$ was estimated from measured $I$ using the calibration curve shown in (b).

Figure 8c shows the fluorescent intensity profile $I(z)$ of the torn sample as the function of the distance from the surface $z$. The damage-zone thickness $h=1090 \mu \mathrm{m}$ is obtained where $I(h)$ is $5 \%$ of $I_{\max }$. Note that the difference of $h$ observed in Figure 5 (1700 $\mu \mathrm{m}$ for torn sample) should be attributed to the difference of gel composition. Using the experimental linear regression for the $I-\varepsilon$ relation of uniaxially stretched sample (Figure 8b), the $I(z)$ profile of the torn sample is converted into $\varepsilon(z)$ profile, and the result is also shown in Figure 8c. The inset letters A, B, C and D in Figure 8c correspond to those shown in Figure 8a. The observed maximum fluorescent intensity $I_{\max }$ near the torn surface almost corresponds to the fluorescent 
intensity $I$ at yielding point under uniaxial stretching. The damage zone thus measured predominantly corresponds to the pre-yielding region with small strain $(0.5<\varepsilon<1.6$ for this gel). Since the yielded region should exist closer to the crack tip, this result implies that the yielded region is close to or less than the spatial resolution of current experiment $(\sim 50 \mu \mathrm{m})$, much narrower than the pre-yielding region $(>1000 \mu \mathrm{m})$. Because the DN gel was not re-swollen in water after tearing, the wide pre-yielding region $(1090 \mu \mathrm{m})$ determined by the fluorescent measurement should be very close to the actual damage-zone thickness at its relaxed state.

Herein, we compare our result to the previous report by Liang et al. on a DN gel using optical microscopes. ${ }^{37}$ In this previous paper, the damage zone of a torn sample in re-swollen state was observed as $\sim 900 \mu \mathrm{m}$, which was assigned, according to their morphological features, as hardened zone $(\sim 100 \mu \mathrm{m})$ near the crack tip, yielded zone $(\sim 200 \mu \mathrm{m})$ in the intermediate region, and pre-yielding zone $(\sim 600 \mu \mathrm{m})$ in the region near the undamaged region. Considering that the re-swelling ratio $q_{\mathrm{r}}$ in stretched direction of a similar DN gel is $q_{\mathrm{r}} \approx 2.0$ in the hardened and yielded region and $q_{\mathrm{r}} \approx 1.2$ in pre-yielded region, ${ }^{35,63,64}$ the damage zone reported by Liang et al. can be re-evaluated in the unswollen state as $\sim 650 \mu \mathrm{m}$, consisting $\sim 50-\mu \mathrm{m}$ hardened zone, 100 $\mu \mathrm{m}$ yielded zone and $\sim 500-\mu \mathrm{m}$ pre-yielding zone. The hardened and yielded zones of approximately $150-\mu \mathrm{m}$-thick near the fractured surface may not be clearly observed in our work because this is closed to the experimental resolution of our current fluorescent method. The thickness of the whole damage zone including pre-yielding region gives similar value as order estimation but some discrepancy ( $\sim 1090 \mu \mathrm{m}$ in our case and $\sim 650-\mu \mathrm{m}$ in sample of Liang et al.). We consider that this is attributed to (1) a small difference of gel composition that results in different damage-zone thickness, (2) difference of sample geometry, i.e., thickness of the gel 
being $3.1 \mathrm{~mm}$ for ours and $0.1 \mathrm{~mm}$ for Liang et al.'s, (3) an error on the re-evaluation to correct the re-swelling degree, and (4) a poor sensitivity of the optical microscope observation in the previous paper which may not observe the region of small damage. In summary, the damagezone thickness determined by the current method is reasonably consistent with that in previous report.

(ii) Stress profile. Similarly, the stress profile $\sigma(z)$ around the crack tip was also obtained from $\varepsilon(z)$ and the stress-strain curve. Figure 9a(i) shows the $\sigma(z)$ profile thus obtained. This $\sigma(z)$ reflects the maximum tensile stress experienced by the crack tip at a position $z$ from the crack plane at relaxed state. Note that the value of $\sigma$ smaller than $0.17 \mathrm{MPa}$ cannot be obtained because it is below the threshold to exhibit fluorescence (see B in Figure 8a). Note that the leg's strain far from the crack was observed as $\varepsilon \approx 0.14$ that is enough below the threshold to show fluorescence.

Here we consider the stress profile under the deformed state. Figures $9 \mathbf{b}(\mathbf{i})$ and $9 \mathbf{b}$ (ii) illustrate the shapes of a sample at undeformed state and deformed state during crack advancing, respectively, using a similar approach in literatures ${ }^{2,33,61}$. In the $\mathrm{xz}$ coordinate that represents the undeformed state, $(x, z)=(0,0)$ is set at the crack tip. We consider two elements positioned far ahead of the crack tip $(x \ll 0)$ at $\mathrm{A}_{0}$ and $\mathrm{B}_{0}$, initially. When the crack advances, the positions of the two elements in relative to the crack tip equivalently move along the red $\left(\mathrm{A}_{0}-\mathrm{A}^{\prime}-\mathrm{A}\right)$ and blue $\left(\mathrm{B}_{0}-\mathrm{B}^{\prime}-\mathrm{B}\right)$ lines, respectively (Figure $9 \mathbf{b}(\mathrm{ii})$ ), where $\mathrm{A}^{\prime}$ and $\mathrm{B}^{\prime}$ are the nearest points to the crack tip for each line (i.e. $x=0$ with different $z$ for undeformed coordinate), and A and B are far behind the crack tip $(x \gg 0)$. The corresponding trajectories in the undeformed coordinate is also shown in Figure 9b(i). It can be assumed that the stress of these elements is maximized at the 
positions $\mathrm{A}^{\prime}$ and $\mathrm{B}^{\prime}$, respectively. Hence, the element at $\mathrm{A}_{0}$ (no stress) experiences a loading to a maximum stress at $\mathrm{A}^{\prime}$ near the crack tip, and then unloading to A (almost no stress). Such loading-unloading cycle is depicted by the red stress-strain cycle in Figure 9b(iii). The corresponding positions $\mathrm{A}_{0}, \mathrm{~A}^{\prime}$, and $\mathrm{A}$ are denoted on the stress-strain curve. The element at $\mathrm{B}_{0}$ experiences the similar loading-unloading cycle but with a smaller maximum stress than that of the element at $\mathrm{A} 0$.

Here, we consider the distance $z^{\prime}$ in the deformed state, from the crack tip $(x=0, z=0)$ to an element with a position in the undeformed coordinate of $(x=0, z)$. Recalling that the strain profile $\varepsilon(z)$ and stress profile $\sigma(z)$ obtained in Figure 8c and Figure 9a correspond to $\varepsilon(x=0, z)$ and $\sigma(x=0, z)$ respectively in this coordinate system for undeformed state, $z^{\prime}$ is related to its position $z$ at relaxed state as,

$$
\frac{\mathrm{d} z^{\prime}}{\mathrm{d} z}=1+\varepsilon(z)
$$

By integrating Eq. (3),

$$
z^{\prime}(z)=\int_{0}^{z}(1+\varepsilon(z)) d z
$$

The physical meaning of Eq. (4) is illustrated in Figure 9c using a discrete element $\Delta z$ for the differentiation $\mathrm{d} z$. Since $\varepsilon(z)$ decreases with the increase of $z$, the element closer to the crack tip is stretched more. Using Eq. (4), the stress profile around the crack tip at deformed state $\sigma\left(z^{\prime}\right)$ is obtained as shown in Figure 9a(ii). The thickness of damage zone at the deformed state, $h^{\prime}$, is more than $2 \mathrm{~mm}$ for this DN gels, which is about two times larger than that at the relaxed state. 
In Figures 9a(i) and 9a(ii), we found that the stress decreases weakly with increasing $z$ in the range of $0<z<400 \mu \mathrm{m}$ ( or $0<z^{\prime}<1000 \mu \mathrm{m}$ in the deformed coordinate), and then decreases with the further increase of $z$, as clearly seen from the insert double-logarithm plots. In the linear elastic fracture mechanics (LEFM), the stress field near the crack scales with the inverse square root of distance $r$ from the crack tip, $\sigma \sim 1 / \sqrt{r}$. On the other hand, for highly deformable nonlinear elastic solids, a reported theory ${ }^{60,61,65}$ predicts that true stress, $\sigma$ true, scales as inverse of the distance (at undeformed coordinate) from the crack, as $\sigma_{\text {true }} \sim 1 / r$ in a region near the crack tip. To compare with the theory, we calculate the true stress as a function of $z$ using $\sigma_{\text {true }}(z)=$ $\sigma(z)(\varepsilon(z)+1)$ under the assumption of incompressibility. As shown in the inset bottom figure in Figure 9a(i), we found a scaling relation $\sigma_{\text {true }} \sim z^{-0.91}$ at the region $z>400 \mu \mathrm{m}$, which is close to the theoretical prediction $\left(\sigma_{\text {true }} \sim z^{-1.0}\right)$. This result suggests that although the energy dissipation takes place even in the pre-yielding zone, this scaling relation of the soft non-linear elastic solid may apply for the stress field around the crack tip. The deviation of the stress from the scaling relation close to the crack tip $(z<400 \mu \mathrm{m})$ implies that the sample is approaching the yielding in this region. 
(a)

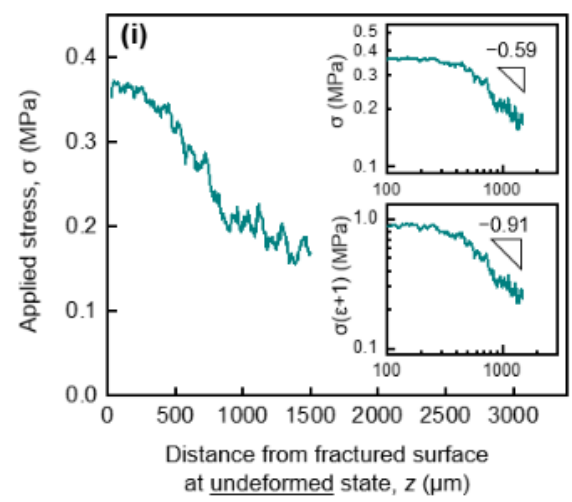

(b) (i) Undeformed state

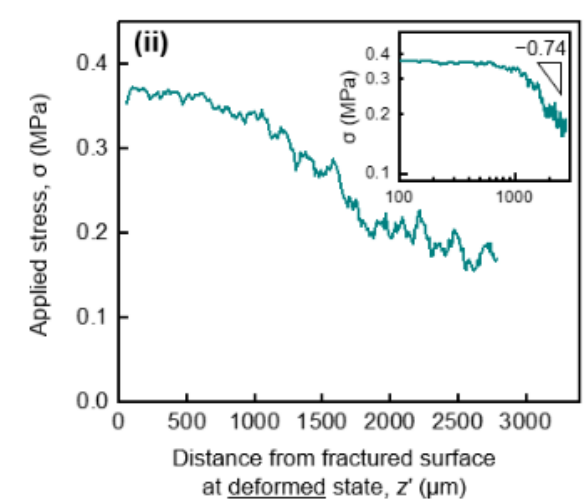

(ii) Deformed state

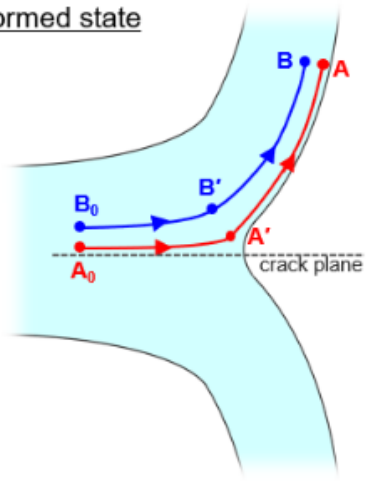

(c)

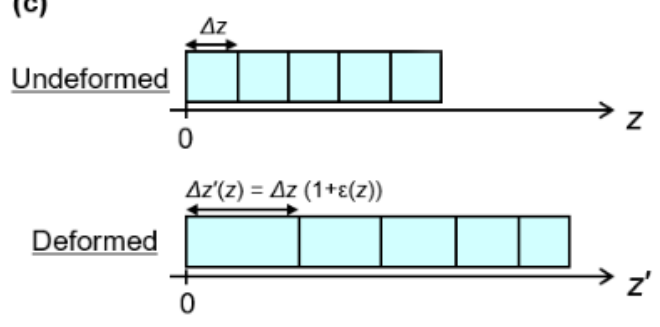

Figure 9. Stress profile around the crack tip estimated from fluorescent observation. (a) The stress profile from the crack plane to the bulk along z-axis at (i) undeformed and (ii) deformed state. Inset figures are the corresponding double-logarithm plots with empirical scaling factor. In the bottom inset figure in (a), the vertical axis is the estimated true stress as $\sigma(z)(\varepsilon(z)+1)$. (b) Mechanical history of elements in the damage zone during the crack propagation. Schematic illustration of a sample at (i) undeformed state and (ii) deformed state during crack propagation. See detailed explanation in the main text. (iii) Schematic stress-strain curves experienced by the two elements initially positioned at $\mathrm{A}_{0}$ and $\mathrm{B}_{0}$ during the crack propagation. (c) Illustration to show that the distance of a point at the crack tip $(x=0, z)$ in deformed state, denoted as $z^{\prime}$, is related to the strain field $\varepsilon(z)$ and $z$ under the assumption of uniaxial deformation (Eq. (4)). 
(iii) Dissipated energy. Finally, we obtain the profile of dissipated energy density $U_{\text {diss }}$ as the function of $z$, and accumulated energy dissipation in the damage zone to discuss its relation with tearing fracture energy $T_{\mathrm{c}}$. To estimate the profile $U_{\text {diss }}(z)$, we conducted cyclic tensile test ${ }^{35}$ to obtain $U_{\text {diss }}-\varepsilon$ relation (Figure 10a), and then obtained $I-U_{\text {diss }}$ relation (Figure 10b) using the $I-\varepsilon$ relation (Figure 8b). Note that $U_{\text {diss }}$ of DN gels is irreversible because it originates from irreversible polymer chain scission. ${ }^{26,27,34,36}$ Fluorescent intensity $I$ is almost proportional to $U_{\text {diss }}$ through the original point. This result confirms that the current method is sensitive enough to characterize the internal fracture even for the small dissipation density. From the $I-U_{\text {diss }}$ relation of the tensile result and the fluorescent profile $I(z)$ of the tearing result (Figure 8c), we obtained the $U_{\text {diss }}(z)$ profile (Figure 10c).

It has been considered that the high fracture toughness of the DN gel is predominantly attributed to the dissipated mechanical energy in the damaged zone, $\Gamma_{\text {diss. }}{ }^{28,29,30,37}$ Previous works simply estimated it by $\Gamma_{\text {diss }}=2 h U_{\text {diss, }}$ under the assumption of a homogeneous dissipated energy density $U_{\text {diss }}$ in the damage zone. The numerical factor 2 is because two symmetric fractured surfaces are formed in fracture test. However, our finding of the fluorescent gradient along the $z$-direction indicates that $U_{\text {diss }}$ is not homogeneous. In such case, $\Gamma_{\text {diss }}$ is estimated by an integral as ${ }^{33}$

$$
\Gamma_{\mathrm{diss}}=2 \int_{0}^{h} U_{\mathrm{diss}}(z) d z
$$

which represents double of the highlighted area in Figure 10c under the $U_{\text {diss }}-z$ curve at $0<z<$ $h$, where $h$ is the damage-zone thickness. Note that the simple estimation, $\Gamma_{\text {diss }}=2 h U_{\text {diss, }}$ is a special case of Eq. (5) when $U_{\text {diss }}$ does not depend on $z$ or $U_{\text {diss }}$ is selected for an appropriate average value. From the integration, the $\Gamma_{\text {diss }}$ of this DN gel is calculated as $298 \mathrm{~J} \mathrm{~m}^{-2}$ (Figure 
10c). The $\Gamma_{\text {diss }}$ thus obtained is in the same order as $T_{\mathrm{c}}\left(906 \mathrm{~J} \mathrm{~m}^{-2}\right)$, but considerably smaller. The possible reasons are discussed in the following section.

(a)

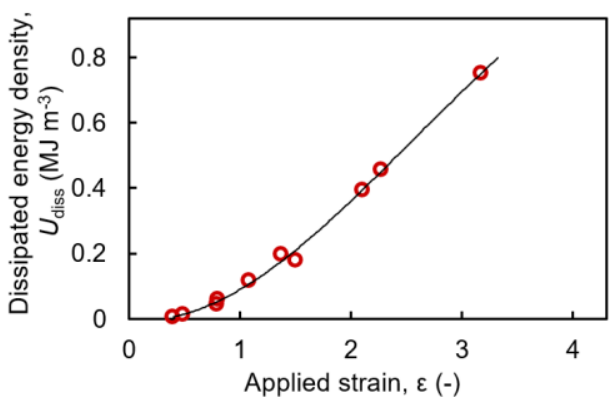

(b)

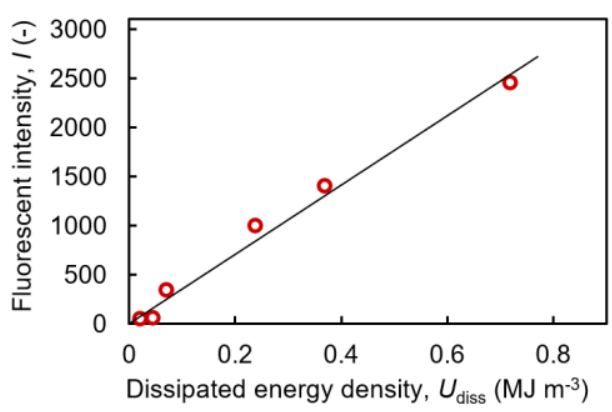

(c)

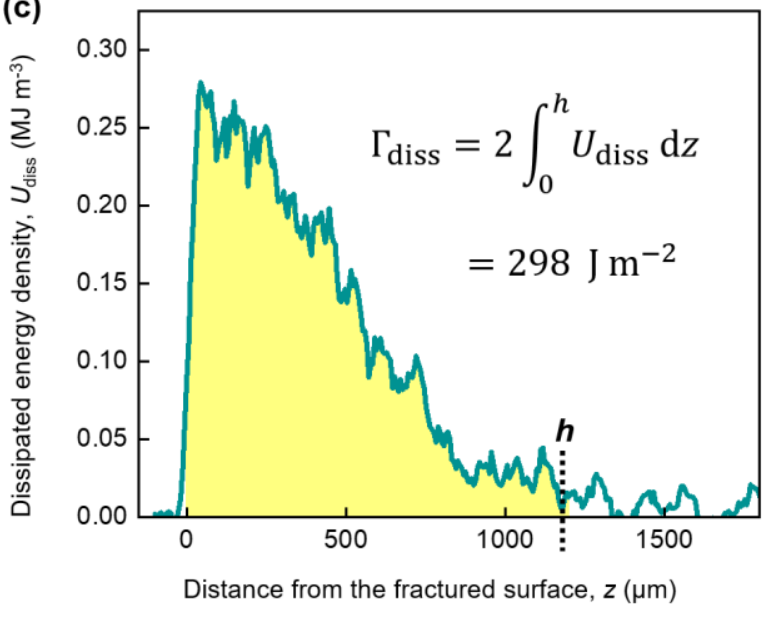

Figure 10. Calculation of the dissipated mechanical energy in the damage zone, $\Gamma_{\text {diss. }}$ (a) Dissipated energy density $U_{\text {diss }}$ as the function of applied strain $\varepsilon$ and (b) fluorescent intensity $I$ as the function of $U_{\text {diss, }}$ which were obtained from uniaxial tensile test. The curves are guide for the eyes. (c) Dissipated energy density profile from the fractured surface to the bulk obtained from the $I-z$ relation (Figure 8c) and the $I-U_{\text {diss }}$ relation (Figure 10b). The fracture energy contributed from the internal fracture, $\Gamma_{\text {diss, }}$, is obtained by integrating $U_{\text {diss }}$ along the $z$ from 0 to $h$ (damage-zone thickness) multiplied by 2 . 


\section{Deviation between $T_{\mathrm{c}}$ and $\Gamma_{\text {diss }}$}

In this section, we discuss the possible reasons of the deviation between measured fracture energy $T_{\mathrm{c}}$ and characterized dissipated energy $\Gamma_{\text {diss. }}$ As described in the introduction, fracture energy of a tough soft material $\Gamma_{\mathrm{c}}\left(\approx T_{\mathrm{c}}\right)$ is characterized as $\Gamma_{\mathrm{c}}=\Gamma_{\text {diss }}+\Gamma_{0}$, where $\Gamma_{0}$ is the intrinsic fracture energy at the crack tip. Theoretical models of the fracture toughness on the DN gel $^{28,29}$ suggested $\Gamma_{\mathrm{c}} \approx \Gamma_{\text {diss, }}$ under the consideration that $\Gamma_{0}$ is negligible. However, from our results $T_{\mathrm{c}}\left(906 \mathrm{~J} \mathrm{~m}^{-2}\right)$ is same order but considerably higher than $\Gamma_{\text {diss }}=298 \mathrm{~J} \mathrm{~m}^{-2}$. We consider that this discrepancy attributes to (1) underestimation of the dissipated energy at yielded zone closed to the fractured surface, (2) non-negligible $\Gamma_{0}$, and (3) other possible errors of this method.

First, we discuss if we underestimated the dissipated energy $\Gamma_{\text {diss. }}$ As mentioned above, the narrow yielded zone (expected as $\sim 100 \mu \mathrm{m}$ ) may not be properly characterized by this method mainly due to limited measurement resolution. In the yielded zone, the dissipated energy density $U_{\text {diss }}$ is expected to be $\sim 1.0 \mathrm{MJ} \mathrm{m}^{-3}$ (see Figure 10a), while we assigned $U_{\text {diss }} \approx 0.25 \mathrm{MJ} \mathrm{m}^{-3}$ from the intensity profile at $0<z<100 \mu \mathrm{m}$. Therefore, accumulated dissipated energy (per unit fractured area) in the yielded zone could be $1.0 \mathrm{MJ} \mathrm{m}^{-3} \times\left(1.0 \times 10^{-4} \mathrm{~m}\right)=100 \mathrm{~J} \mathrm{~m}^{-2}$, while we assigned $\sim 25 \mathrm{~J} \mathrm{~m}^{-2}$ at $0<z<100 \mu \mathrm{m}$. Therefore, $\sim 75 \mathrm{~J} \mathrm{~m}^{-2}$ under estimation is possible. However, it hardly grantees the discrepancy between $T_{\mathrm{c}}$ and $\Gamma_{\text {diss. }}$

Next, we discuss the intrinsic fracture energy $\Gamma_{0}$. Previously, $\Gamma_{0}$ is considered identical to the fracture energy of the pure second network, and it has been assumed to be negligible because some chemically crosslinked PAAm gels reported have a fracture energy $\Gamma_{2 \mathrm{nd}} \sim 10 \mathrm{~J} \mathrm{~m}^{-2}$ that is 
much smaller than the fracture energy of DN gel. ${ }^{28,29,66}$ However, Suo and co-workers recently reported that the fracture energy of some loosely-crosslinked PAAm gels, which is similar as the second network of a DN gel in usual case, is $100-500 \mathrm{~J} \mathrm{~m}^{-2} .4,18,67-69$ Therefore, the $\Gamma_{2 \text { nd }}$ may not be negligible. Fatigue threshold, which is often regarded as intrinsic fracture energy of soft materials, of a DN gel is also found as high as $\sim 400 \mathrm{~J} \mathrm{~m}^{-2} \cdot{ }^{25}$ Moreover, it is still an open question whether the intrinsic fracture energy $\Gamma_{0}$ can be approximated as the $\Gamma_{2 \mathrm{nd}}$.

To re-examine $\Gamma 0$, we measured the fracture energy of a PAAm gel (the second network) prepared at the same formulation (AAm 4.0 $\mathrm{M}$ as monomer and MBAA $0.8 \mathrm{mM}$ as crosslinker) as that of the second network of the DN gel used here. We also measured the fracture energy of the DN gel that had been experienced a prestretching close to its breaking (see Supporting Note and Figures S5-S7 for detail). Since we used the single-edge notched fracture test ${ }^{56,70}$ on these samples for convenience, we denote the fracture energy as $G_{\mathrm{c}}$ to distinguish the tearing fracture energy $T_{\mathrm{c}}$. The results are shown in Figure 11. The $G_{\mathrm{c}}$ of the virgin (unstretched) DN gel is determined as $830 \mathrm{~J} \mathrm{~m}^{-2}$ that is close to $T_{\mathrm{c}}\left(906 \mathrm{~J} \mathrm{~m}^{-2}\right)$. The $G_{\mathrm{c}}$ of the PAAm gel at its aspolymerized state, with polymer weight fraction of $28 \mathrm{wt} . \%$, was determined as $320 \mathrm{~J} \mathrm{~m}^{-2}$. In the DN gel, however, the PAAm second-network concentration is $17 \mathrm{wt} . \%$ because the DN gel was swollen in water after the second-network polymerization. Knowing that the fracture energy of a simple elastic hydrogel upon swelling linearly decreases with the decrease of the polymer concentration, ${ }^{1,59} G_{\mathrm{c}}$ of the PAAm second network at $17 \mathrm{wt} . \%$ is estimated as $190 \mathrm{~J} \mathrm{~m}^{-2}$. Therefore, $\Gamma_{2 \text { nd }}$ is not negligibly small compared to the fracture energy of the DN gel. To measure the fracture energy of the largely-stretched DN gel, $G_{\mathrm{c}}{ }^{*}$, as an approximation of the $\Gamma_{0},{ }^{71}$ a DN gel was first stretched close to the strain at break to induce internal fracturing as possible, and then the sample was unloaded to original length. The single-edge notched fracture test was 
carried out on this prestretched sample by making a notch vertical to the prestretched direction (Figure 11a). Surprisingly, we found $G_{\mathrm{c}}{ }^{*}=700 \mathrm{~J} \mathrm{~m}^{-2}$ that is much higher than $\Gamma_{2 \text { nd }}$ (Figure 11b). This is also supported by the raw $\sigma-\lambda$ curves of the notched samples for these two gels. As shown in Figure 11c, the curves of the two notched gels until its rupture is similar but the rupture point is significantly different, indicating the large difference of the fracture energy. Although $G_{c}{ }^{*}$ thus measured should be slightly higher than the $\Gamma_{0}$ because some of the first network strands should further break with the further increase of stretch even in this largelystretched DN gel, the results suggest that $\Gamma_{0}$ is higher than $\Gamma_{2 \text { nd. }}$. Moreover, the results in Figure 11b imply that $\Gamma_{0}$ might be not far from $\Gamma_{\mathrm{c}}$ itself. 


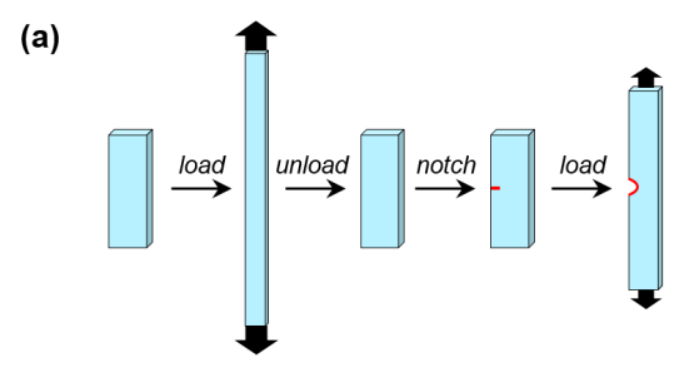
(c) - - - Virgin DN gel, unnotched — Virgin DN gel, notched
- - - Stretched DN gel, unnotched — Stretched DN gel, notched
- - - PAAm gel, unnotched _ PAAm gel, notched
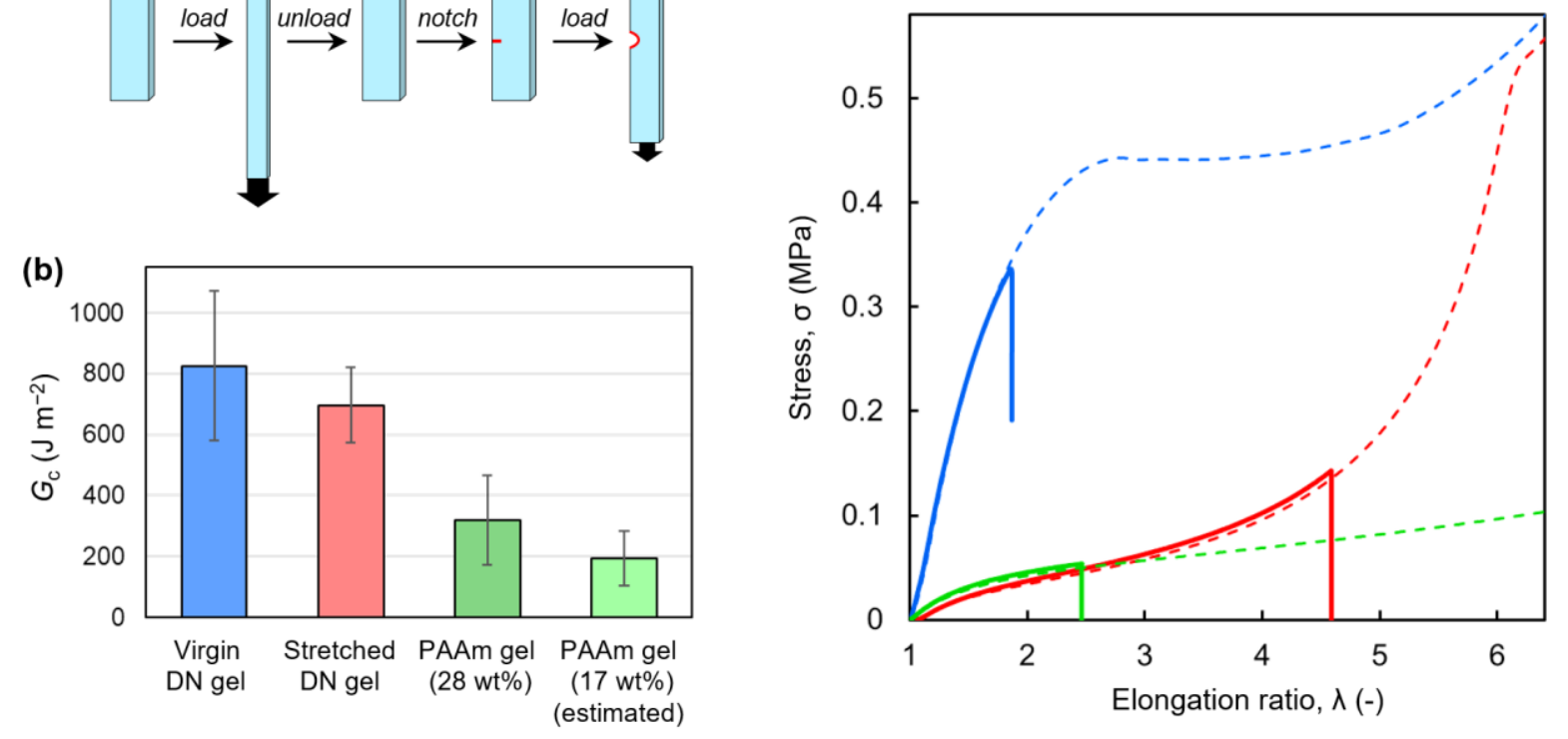

Figure 11. Fracture energy, $G_{c}$, of various gels measured by single-edge notch test. (a) Schematic illustration of the procedures for measuring the fracture energy of a DN gel with significant internal damage. A virgin DN gel is stretched to a strain $\varepsilon$ near its rupture point and then the fracture energy of the stretched gel is measured by the single-edge notch test. (b) Fracture energy $G_{\mathrm{c}}$ of the virgin DN gel, stretched $(\varepsilon=6) \mathrm{DN}$ gel, and the PAAm gel with polymer weight fraction of 28 wt.\%. The estimated fracture energy of a PAAm gel with a polymer weight fraction of $17 \mathrm{wt} . \%$, the same as that in the DN gel, is also shown. The error bars represent the standard deviation for 3-4 measurements. (c) Typical stress-elongation ratio curves of the notched (solid curves) and unnotched (dashed curves) samples of the virgin DN gel (blue), stretched $(\varepsilon=6) \mathrm{DN}$ gel (red), and the PAAm gel with polymer weight fraction of $28 \mathrm{wt} . \%$ (green). To highlight the results of notched samples, only parts of the curves are shown for the unnotched samples. The experimental detail including the whole curves is found in Supplementary Information. 
Finally, we discuss other possible errors of this method for obtaining the stress-strain field and accumulated energy dissipation $\Gamma_{\text {diss. }}$ First, $I-U_{\text {diss }}$ relations between uniaxial stretching and tearing may not exactly be identical. The actual deformation around the crack tip is more complicated, which may cause different $I-U_{\text {diss }}$ relations between uniaxial stretching and tearing. Strain rate may also affect the $I-U_{\text {diss }}$ relations. The strain rate around the crack tip under the tearing is significantly higher than that of uniaxial tensile test. Therefore, the rate of mechanoradical generation is different, which might result in difference in NIPPAm polymerization efficiency. Second, we assumed that $U_{\text {diss }}$ does not vary along the sample thickness direction (y-direction, see Figure 4). If actual $U_{\text {diss }}$ varies with $y, \Gamma_{\text {diss }}$ calculated using Eq. (5) induces errors. Third, water migration might exist. We indicated that re-swelling treatment is not required in this method so that the damage-zone thickness characterized is very closed to the fractured state. Indeed, we did not observe a difference in sample thickness between the damaged and un-damaged region with our eyes. Even so, some water migration from the undamaged region to the damaged region may occur due to difference in the osmotic pressure between the two regions, which can dilute the PNIPAAm in the damage zone and therefore reduce the fluorescent intensity. In addition, it should be also mentioned that the mechanoradical polymerization is sensitive to oxygen dissolved in the gel since oxygen quenches radicals. In our experiment, we performed the mechanoradical polymerization in a glove box filled with argon, where oxygen concentration typically ranged around $10-50 \mathrm{ppm}$. The concentration difference among the experiments may affect the monomer conversion, resulting in deviation in fluorescent intensity.

Even taking such uncertain experimental errors, the series of the discussion above brings a new important insight on the toughness of a DN gels. That is, the dissipation in the pre-yielding zone 
and intrinsic fracture energy ahead of a crack tip make a large contribution to $\Gamma_{\text {diss, }}$ as well as the dissipation in the yielded zone. Previously, most of researches on a DN gel focused on energy dissipation only in yielded zone because the energy dissipation density $\left(\mathrm{J} \mathrm{m}^{-3}\right)$ in the pre-yielding zone is relatively small so that it has been considered negligible. ${ }^{28,29,31,37}$ However, our results by proposed method indicate that $\Gamma_{\text {diss }}$ in the pre-yielded zone is comparable or possibly even larger than the $\Gamma_{\text {diss }}$ in the yielding zone. This is because dissipation volume $\left(\mathrm{m}^{3}\right)$ in the pre-yielding zone is significantly larger than that of yielded zone, even though dissipation density $\left(\mathrm{J} \mathrm{m}^{-3}\right)$ is relatively small. Specifically, in pre-yielding region the dissipated energy density per unit volume is $\sim 10$ times smaller and the dissipated volume is $\sim 10$ times larger than those in yielded zone, so that the two zones make comparable contributions to $\Gamma_{\text {diss. }}$ Besides, we also found from additional experiments that $\Gamma_{0}$ is not negligibly small as $\sim 10^{1} \mathrm{~J} \mathrm{~m}^{-2}$ that had been predicted but has certain level of contribution in the order of $\sim 10^{2} \mathrm{~J} \mathrm{~m}^{-2}$. Our data further implies that the intrinsic fracture energy is higher than the fracture energy of the second network, which should be further investigated in future works. At present, taking considerations of experimental errors, we conclude that the fracture energy of the $\mathrm{DN}$ gel is contributed by $\Gamma_{\text {diss }}$ in yielded zone of $50-300 \mathrm{~J} \mathrm{~m}^{-2}, \Gamma_{\text {diss }}$ in pre-yielding zone of $200-400 \mathrm{~J} \mathrm{~m}^{-2}$, and intrinsic fracture energy $\Gamma_{0}$ of $200-600 \mathrm{~J} \mathrm{~m}^{-2}$, resulting in $T_{\mathrm{c}} \approx 900 \mathrm{~J} \mathrm{~m}^{-2}$. 


\section{CONCLUSION}

We developed the new method to visualize the submillimeter-scale damage zone of DN gels around the crack tip by combining the mechanoradical polymerization of NIPAAm, fluorescent

probe ANS, and a laser scanning confocal microscope (LSCM). Because this method records the internal fracturing in three-dimensions, it can visualize the mechanical history of complicated deformation and internal damage. Besides, this technique can also record high-speed fracture in principle, which is often difficult to be captured by real-time observation. The advantage of this method is no need of mechanophore specifically incorporated into the polymer. It is also potentially applicable to materials having dissipation mechanism based on homolytic bond scission, as demonstrated by the fluorescent images on the fractured surface of single-network hydrogels.

With this method, the degree of internal fracturing in the damage zone of DN gels have been quantitatively characterized to estimate the maximum stress, strain, and dissipated mechanical energy density. Our results suggest that the dissipated mechanical energy in pre-yielding zone with certain internal fracturing is as important as that in yielded zone because the dissipating volume of the pre-yielding zone is significantly larger than the yielded zone. In addition to the energy dissipation in the damage zone $\Gamma_{\text {diss, }}$, the intrinsic fracture energy ahead of the crack tip $\Gamma_{0}$ is also found to be higher than expected in previous researches on DN gels. Further precise quantitative analysis is required for clarifying the toughening mechanism of the DN gels. 


\section{ASSOCIATED CONTENT}

\section{Supporting Information}

The following files are available.

Figures S1-S7 and a Supporting Note (PDF)

\section{AUTHOR INFORMATION}

\section{Corresponding Author}

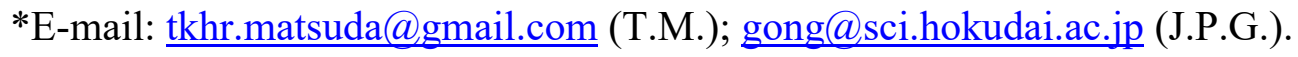

\section{Author Contributions}

\#These authors contributed equally.

\section{Note}

The authors declare no competing financial interest.

\section{ACKNOWLEDGMENT}

This research was partially funded by Grant-in-Aid for JSPS Research Fellow (No. 17J09290) and for Scientific Research (S) (No. 17H06144) from the Japan Society for the Promotion of Science, and by the ImPACT Program of the Council for Science, Technology and Innovation (Cabinet Office, Government of Japan). The authors thank Toagosei Co., Ltd. for providing AMPS and NaAMPS. R.K. and T.M. thank the Nikon Imaging Center at Hokkaido University 
and Kentaro Kobayashi for being very helpful with confocal microscopy, image acquisition, and analysis. T.M. thanks Yukiko Hane to perform single-edge notch fracture tests.

\section{REFERENCES}

(1) Creton, C. 50th anniversary perspective: Networks and gels: Soft but dynamic and tough. Macromolecules 2014, 47 (19), 6783-6790.

(2) Long, R.; Hui, C.-Y. Fracture toughness of hydrogels: Measurement and interpretation. Soft Matter 2016, 12 (39), 8069-8086.

(3) Fan, H.; Gong, J. P. Fabrication of bioinspired hydrogels: Challenges and opportunities. Macromolecules 2020, 53 (8), 2769-2782.

(4) Bai, R.; Yang, J.; Suo, Z. Fatigue of hydrogels. Eur. J. Mech. A Solids 2019, 74, 337-370.

(5) Grijalvo, S.; Eritja, S.; Díaz, D. D. On the race for more stretchable and tough hydrogels. Gels 2019, 5, 24.

(6) Fuchs, S.; Shariati, K.; Ma, M. Specialty tough hydrogels and their biomedical applications. Adv. Healthcare Mater. 2020, 9, 1901396.

(7) Annabi, N.; Tamayol, A.; Uquillas, J. A.; Akbari, M.; Bertassoni, L. E. Cha, C.; CamciUnal, G.; Dokmeci, M. R. Peppas, N. A.; Khademhosseini, A. 25th anniversary article: Rational design and applications of hydrogels in regenerative medicine. Adv. Mater. 2014, $26,85-124$.

(8) Rus, D.; Tolley, M. Design, fabrication and control of soft robots. Nature 2015, 521, 467475.

(9) Polygerinos, P.; Correll, N.; Morin, A. A.; Mosadegh, B.; Onal, C. D.; Petersen, K.; Cianchetti, M.; Tolley, M. T.; Shepherd, R. F. Soft robotics: Review of fluid-driven 
intrinsically soft devices; manufacturing, sensing, control, and applications in human-robot interaction. Adv. Eng. Mater. 2017, 19, 1700016.

(10)Rogers, J. A.; Someya, T.; Huang, Y. Materials and mechanics for stretchable electronics. Science 2010, 327, 1603-1607.

(11) Lim, H.-R.; Kim, H. S.; Qazi, R.; Kwon, Y. T.; Jeong, J.-W. Yeo, W.-H. Advanced soft materials, sensor integrations, and applications of wearable flexible hybrid electronics in healthcare, energy, and environment. Adv. Mater. 2020, 32, 1901924.

(12)Creton, C.; Ciccotti, M. Fracture and adhesion of soft materials. Rep. Prog. Phys. 2016, 79 (4), 046601.

(13)Zhao, X. Multi-scale multi-mechanism design of tough hydrogels: Building dissipation into stretchy networks. Soft Matter 2014, 10 (5), 672-687.

(14)Gong, J. P.; Katsuyama, Y.; Kurokawa, T.; Osada, Y. Double-network hydrogels with extremely high mechanical strength. Adv. Mater. 2003, 15, 1155-1158.

(15)Gong, J. P. Why are double network hydrogels so tough? Soft Matter 2010, 6, 2583-2590.

(16)Chen, Q.; Chen, H.; Zhu, L.; Zheng, J. Fundamentals of double network hydrogels. J. Mater. Chem. B 2015, 3, 3654-3676.

(17) Nakajima, T. Generalization of the sacrificial bond principle for gel and elastomer toughening. Polym. J. 2017, 49, (6), 477-485.

(18)Sun, J.-Y.; Zhao, X.; Illeperuma, W. R. K.; Chaudhuri, O.; Oh, K. H.; Mooney, D. J.; Vlassak, J. J.; Suo, Z. Highly stretchable and tough hydrogels. Nature 2012, 489, 133-136.

(19)Ducrot, E.; Chen, Y.; Bulters, M.; Sijbesma, R. P.; Creton, C. Toughening elastomers with sacrificial bonds and watching them break. Science 2014, 344, 186-189.

(20)Matsuda, T.; Nakajima, T.; Gong, J. P. Fabrication of tough and stretchable hybrid doublenetwork elastomers using ionic dissociation of polyelectrolyte in nonaqueous media. Chem. Mater. 2019, 31, 3766-3776. 
(21)Ahmed, S.; Nakajima, T.; Kurokawa, T.; Anamul Haque, M.; Gong, J. P. Brittle-ductile transition of double network hydrogels: Mechanical balance of two networks as the key factor. Polymer 2014, 55 (3), 914-923.

(22)Frauenlob, M.; King, D. R.; Guo, H.; Ishihara, S.; Tsuda, M.; Kurokawa, T.; Haga, H.; Tanaka, S.; Gong, J. P. Modulation and characterization of the double network hydrogel surface-bulk transition. Macromolecules 2019, 52, 6704-6713.

(23)Tanaka, Y.; Kuwabara, R.; Na, Y.-H.; Kurokawa, T.; Gong, J. P.; Osada, Y. Determination of fracture energy of high strength double network hydrogels. J. Phys. Chem. B 2005, 109, 11559-11562.

(24)Nakajima, T.; Furukawa, H.; Tanaka, Y.; Kurokawa, T.; Gong, J. P. Effect of void structure on the toughness of double network hydrogels. J. Polym. Sci. B. Polym. Phys. 2011, 49, 1246-1254.

(25)Zhang, W.; Liu, X.; Wang, J.; Tang, J.; Hu, J.; Lu, T.; Suo, Z. Fatigue of double-network hydrogels. Eng. Fract. Mech. 2018, 187, 74-93.

(26)Na, Y.-H.; Tanaka, Y.; Kawauchi, Y.; Furukawa, H.; Sumiyoshi, T.; Gong, J. P.; Osada, Y. Necking phenomenon of double-network gels. Macromolecules 2006, 39, 4641-4645.

(27)Webber, R. E.; Creton, C.; Brown, H. R.; Gong, J. P. Large strain hysteresis and Mullins effect of tough double-network hydrogels. Macromolecules 2007, 40, 2919-2927.

(28)Brown, H. R. A model of the fracture of double network gels. Macromolecules 2007, 40 (10), 3815-3818.

(29)Tanaka, Y. A local damage model for anomalous high toughness of double-network gels. Europhys. Lett. 2007, 78 (5), 56005.

(30)Tanaka, Y.; Kawauchi, Y.; Kurokawa, T.; Furukawa, H.; Okajima, T.; Gong, J. P. Localized yielding around crack tips of double-network gels. Macromol. Rapid Commun. 2008, 29 (18), 1514-1520. 
(31)Yu, Q. M.; Tanaka, Y.; Furukawa, H.; Kurokawa, T.; Gong, J. P. Direct observation of damage zone around crack tips in double-network gels. Macromolecules 2009, 42 (12), $3852-3855$.

(32)Kolvin, I.; Kolinski, J. M.; Gong, J. P.; Fineberg, J. How supertough gels break. Phys. Rev. Lett. 2018, 121, 135501.

(33)Qi, Y.; Caillard, J.; Long, R. Fracture toughness of soft materials with rate-independent hysteresis. J. Mech. Phys. Solids 2018, 118, 341-364.

(34)Matsuda, T.; Nakajima, T.; Fukuda, Y.; Hong, W.; Sakai, T.; Kurokawa, T.; Chung, U.-I.; Gong, J. P. Yielding criteria of double network hydrogels. Macromolecules 2016, 49 (5), $1865-1872$.

(35)Nakajima, T.; Kurokawa, T.; Ahmed, S.; Wu, W.-L.; Gong, J. P. Characterization of internal fracture process of double network hydrogels under uniaxial elongation. Soft Matter 2013, 9 , 1955-1966.

(36)Matsuda, T.; Kawakami, R.; Namba, R.; Nakajima, T.; Gong, J. P. Mechanoresponsive selfgrowing hydrogels inspired by muscle training. Science 2019, 363, 504-508.

(37)Liang, S.; Wu, Z. L.; Hu, J.; Kurokawa, T.; Yu, Q. M.; Gong, J. P. Direct observation on the surface fracture of ultrathin film double-network hydrogels. Macromolecules 2011, 44, 3016-3020.

(38)Akbulatov, S.; Boulatov, R. Experimental polymer mechanochemistry and its interpretational frameworks. ChemPhysChem 2017, 18, 1422-1450.

(39)Do, J.-L.; Friščić, T. Chemistry 2.0: Developing a new, solvent-free system of chemical synthesis based on mechanochemistry. Synlett 2017, 28, 2066-2092.

(40)Li, J.; Nagamani, C.; Moore, J. S. Polymer mechanochemistry: From destructive to productive. Acc. Chem. Res. 2015, 48, 2181-2190. 
(41)Ribas-Arino, J.; Marx, D. Covalent mechanochemistry: Theoretical concepts and computational tools with applications to molecular nanomechanics. Chem. Rev. 2012, 112, 5412-5487.

(42)Stratigaki, M.; Göstl, R. Methods for exerting and sensing force in polymer materials using mechanophores. ChemPlusChem 2020, 85, 1-10.

(43)Celestine, A.-D. N.; Beiermann, B. A.; May, P. A.; Moore, J. S.; Sottos, N. R.; White, S. R. Fracture-induced activation in mechanophore-linked, rubber toughened PMMA. Polymer 2014, 55, 4164-4171.

(44)Wang, Q.; Gossweiler, G. R.; Craig, S. L.; Zhao, X. Mechanics of mechanochemically responsive elastomers. J. Mech. Phys. Solids 2015, 82, 320-344.

(45)Clough, J. M.; Creton, C.; Craig, S. L.; Sijbesma, R. P. Covalent bond scission in the mullins effect of a filled elastomer: Real-time visualization with mechanoluminescence. Adv. Funct. Mater. 2016, 26, 9063-9074.

(46)Millereau, P.; Ducrot, E.; Clough, J. M.; Wiseman, M. E.; Brown, H. R.; Sijbesma, R. P.; Creton, C. Mechanics of elastomeric molecular composites. Proc. Natl. Acad. Sci. U.S.A. 2018, 115 (37) 9110-9115.

(47)Celestine, A.-D. N.; Sottos, N. R.; White, S. R. Strain and stress mapping by mechanochemical activation of spiropyran in poly(methyl methacrylate). Strain 2019, 55, e12310.

(48)Zhang, H.; Zeng, D.; Pan, Y.; Chen, Y.; Ruan, Y.; Xu, Y.; Boulatov, R.; Creton, C.; Weng, W. Mechanochromism and optical remodeling of multi-network elastomers containing anthracene dimers. Chem. Sci. 2019, 10, 8367-8373.

(49)Kim, T. A.; Lamuta, C.; Kim, H.; Leal, C.; Sottos, N. R. Interfacial force-focusing effect in mechanophore-linked nanocomposites. Adv. Sci. 2020, 1903464.

(50)Stratigaki, M.; Baumann, C.; van Breemen, L. C. A.; Heuts, J. P. A.; Sijbesma, R. P.; Göstl, R. Fractography of poly( $N$-isopropylacrylamide) hydrogel networks crosslinked with 
mechanofluorophores using confocal laser scanning microscopy. Polym. Chem. 2020, 11, $358-366$.

(51)Lin, Y.; Barbee, M. H.; Chang, C.-C.; Craig, S. L. Regiochemical effects on mechanophore activation in bulk materials. J. Am. Chem. Soc. 2018, 140, 15969-15975.

(52)Chen, Y.; Yeh, C. J.; Qi, Y.; Long, R.; Creton, C. From force-responsive molecules to quantifying and mapping stresses in soft materials. Sci. Adv. 2020, 6, eaaz5093.

(53) Schild, H. G. Poly( $N$-isopropylacrylamide): Experiment, theory and application. Prog. Polym. Sci., 1992, 17, 163-249.

(54)Turner, D. C.; Brand, L. Quantitative estimation of protein binding site polarity. Fluorescence of $N$-arylaminonaphthalenesulfonates. Biochemistry 1968, 7, 3381-3390.

(55)Ito, D.; Itagaki, H. Clarification of the inner microenvironments in poly $(\mathrm{N}$ isopropylacrylamide) hydrogels in macrogel and microgel forms using a fluorescent probe technique. Eur. Polym. J. 2018, 99, 277-283.

(56)Rivlin, R. S.; Thomas, A. G. Rupture of rubber. I. Characteristic energy for tearing. J. Polym. Sci. 1953, 10, 291-318.

(57)Greensmith, H. W.; Thomas, A. G. Rupture of rubber. III. Determination of tear properties. J. Polym. Sci. 1955, 18, 189-200.

(58)Lake, G. J.; Thomas, A. G. The strength of highly elastic materials. Proc. R. Soc. London, Ser. A 1967, A300, 108-119.

(59)Akagi, Y.; Sakurai, H.; Gong, J. P.; Chung, U.-I.; Sakai, T. Fracture energy of polymer gels with controlled network structures. J. Chem. Phys. 2013, 139 (14), 144905.

(60)Long, R.; Hui, C. Y. Crack tip fields in soft elastic solids subjected to large quasi-static deformation - A review. Extreme Mech. Lett. 2015, 4, 131-155. 
(61)Long, R.; Hui, C.-Y.; Gong, J. P.; Bouchbinder, E. The fracture of highly deformable soft materials: A tale of two length scales. Annual Rev. Cond. Matter Phys., 2020 Accepted. Preprint: [arXiv:2004.03159]

(62)Mai, T.-T.; Matsuda, T.; Nakajima, T.; Gong, J. P.; Urayama, K. Distinctive characteristics of internal fracture in tough double network hydrogels revealed by various modes of stretching, Macromolecules 2018, 51(14), 5245-5257.

(63)Mai, T.-T.; Matsuda, T.; Nakajima, T.; Gong, J. P.; Urayama, K. Damage cross-effect and anisotropy in tough double network hydrogels revealed by biaxial stretching. Soft Matter 2019, 15, 3719-3732.

(64)Guo, H.; Hong, W.; Kurokawa, T.; Matsuda, T.; Wu, Z. L. Nakajima, T.; Takahata, M.; Sun, T.; Rao, P.; Gong, J. P. Internal damage evolution in double-network hydrogels studied by microelectrode technique. Macromolecules 2019, 52, 7114-7122.

(65) Stephenson, R. A. The equilibrium field near the tip of a crack for finite plane strain of incompressible elastic materials. J. Elast. 1982, 12, 65-99.

(66) Tanaka, Y,; Fukao, K.; Miyamoto, Y. Fracture energy of gels. Eur. Phys. J. E 2000, 3, 395401.

(67)Zhang, E.; Bai, R. Morelle, X. P.; Suo, Z. Fatigue fracture of nearly elastic hydrogels. Soft Matter 2018, 14, 3563-3571.

(68) Yang, C.; Yin, T.; Suo, Z. Polyacrylamide hydrogels. I. Network imperfection. J. Mech. Phys. Solids 2019, 131, 43-55.

(69)Liu, J.; Yang, C.; Yin, T.; Wang, Z.; Qu, S.; Suo, Z. Polyacrylamide hydrogels. II. Elastic dissipater. J. Mech. Phys. Solids 2019, 133, 103737.

(70)Greensmith, H. W. Rupture of rubber. X. The change in stored energy on making a small cut in a test piece held in simple extension. J. Appl. Polym. Sci. 1963, 7, 993-1002. 
(71)Zhang, T.; Lin, S.; Yuk, H.; Zhao, X. Predicting fracture energies and crack-tip fields of soft tough materials. Extreme Mech. Lett. 2015, 4, 1-8. 